\title{
Chromium-Mediated Synthesis of Polycyclic Aromatic Compounds from Halobiaryls
}

Ken-ichiro Kanno, Yuanhong Liu, Atsushi lesato, Kiyohiko Nakajima ${ }^{\dagger}$, and Tamotsu Takahashi*

Catalysis Research Center, and Graduate School of Pharmaceutical Sciences, Hokkaido University, and SORST, Japan Science and Technology Agency (JST), Sapporo 001-0021, Japan; 'Department of Chemistry, Aichi University of Education, Igaya, Kariya, Aichi 448-8542, Japan

tamotsu@cat.hokudai.ac.jp

\section{Contents}

1) General information and detailed synthetic procedures

S2-S5

2) Tables of X-ray analysis of 3,4-diethyl-8,9-diphenyl-9b-azacyclopenta[cd]phenalene (12b)

S6-S7

3) NMR spectra

S8-S28 
General Information. All reactions were carried out with standard Schlenk techniques under nitrogen. Tetrahydrofuran, hexane were distilled over sodium and benzophenone. Chromium(III) chloride was purchased from Soekawa Rikagaku Co. Ltd. 2,2-Dibromobiphenyl, ${ }^{1}$ 2-bromobiphenyl, ${ }^{2}$ 2-iodo-4'-methylbiphenyl, ${ }^{3}$ and 1-(2-iodophenyl)naphthalene ${ }^{2}$ were prepared according to the literatures. All other reagents were commercially available and used without further purification. ${ }^{1} \mathrm{H}$ and ${ }^{13} \mathrm{C}$ NMR spectra were recorded in $\mathrm{CDCl}_{3}$ or benzene- $d_{6}$ (containing 1\% TMS) solutions on a Bruker-ARX400 or JEOL JNM-AL300 NMR spectrometers. GC analyses were performed on SHIMADZU GC-14A equipped with fused silica capillary column SHIMADZU CBP1-M25-025 and SHIMADZU C-R6A-Chromatopac integrator.

General Procedure for the Synthesis of Phenanthrene Derivatives using Chromium(III) Chloride: To a solution of 2,2'-dibromobiphenyl $1(312 \mathrm{mg}, 1.0 \mathrm{mmol})$ in $\mathrm{THF}$ at $-78^{\circ} \mathrm{C}, n$-butyllithium $(1.28 \mathrm{~mL}, 1.56 \mathrm{M}, 2.0 \mathrm{mmol}) \mathrm{was}$ added and stirred for $1 \mathrm{~h}$. Then the mixture was added with chromium(III) chloride (158 mg, $1.0 \mathrm{mmol})$ and warmed to room temperature. After $1 \mathrm{~h}$, an alkyne $(2 \mathrm{mmol})$ was added and the mixture was heated to $50{ }^{\circ} \mathrm{C}$. The mixture was quenched by addition of $3 \mathrm{~N}$ HClaq. and extracted with ethyl acetate. The organic layer was separated, dried with magnesium sulfate, and concentrated in vacuo. The residue was purified by column chromatography on silica gel to afford the corresponding phenanthrene.

9,10-Diethylphenanthrene (2a): This compound was prepared from 2,2'-dibromobiphenyl and 3-hexyne. GC yield: $95 \%$, Isolated yield: $75 \%$, colorless solid, $\mathrm{mp}=107-109{ }^{\circ} \mathrm{C}$ (lit. $\left.{ }^{4} 105-107{ }^{\circ} \mathrm{C}\right) ;{ }^{1} \mathrm{H} \mathrm{NMR}\left(400 \mathrm{MHz}, \mathrm{CDCl}_{3}\right): \delta 1.44(\mathrm{t}$, $J=7.5 \mathrm{~Hz}, 6 \mathrm{H}), 3.28(\mathrm{q}, J=7.6 \mathrm{~Hz}, 4 \mathrm{H}), 7.63-7.70(\mathrm{~m}, 4 \mathrm{H}), 8.19(\mathrm{~d}, J=8.5 \mathrm{~Hz}, 2 \mathrm{H}), 8.77(\mathrm{~d}, J=7.2 \mathrm{~Hz}, 2 \mathrm{H}) ;{ }^{13} \mathrm{C}$ NMR $\left(100 \mathrm{MHz}, \mathrm{CDCl}_{3}\right): \delta 15.37,22.45,123.31,124.91,125.72,126.91,130.21,131.41,135.15$; HRMS calcd for $\mathrm{C}_{18} \mathrm{H}_{18}: 234.1409$, found: 234.1420 .

9,10-Diphenylphenanthrene (2b): This compound was prepared from 2,2'-dibromobiphenyl and diphenylacetylene. Isolated yield: $48 \%$, colorless solid, $\mathrm{mp}=236-237{ }^{\circ} \mathrm{C}$ (lit. $\left.{ }^{5} \quad 238.5-239.0{ }^{\circ} \mathrm{C}\right) ;{ }^{1} \mathrm{H}$ NMR $\left(400 \mathrm{MHz}, \mathrm{CDCl}_{3}\right): \delta 7.14-7.25$ $(\mathrm{m}, 10 \mathrm{H}), 7.46-7.50(\mathrm{~m}, 2 \mathrm{H}), 7.55-7.57(\mathrm{~m}, 2 \mathrm{H}), 7.64-7.68(\mathrm{~m}, 2 \mathrm{H}), 8.80(\mathrm{~d}, J=8.3 \mathrm{~Hz}, 2 \mathrm{H}) ;{ }^{13} \mathrm{C} \mathrm{NMR}(100 \mathrm{MHz}$, $\left.\mathrm{CDCl}_{3}\right): \delta 122.83,126.73,126.81,126.96,127.92,128.18,130.32,131.38,132.21,137.53,139.90$; HRMS calcd for $\mathrm{C}_{24} \mathrm{H}_{18}: 330.1409$, found: 330.1420 .

9-Methyl-10-phenylphenanthrene (2c): This compound was prepared from 2,2'-dibromobiphenyl and 1-phenyl-1-propyne. $\mathrm{GC}$ yield: $75 \%$, Isolated yield: $58 \%$, colorless solid, mp $=100-101{ }^{\circ} \mathrm{C}\left(\right.$ lit. $\left.{ }^{6} 99-100{ }^{\circ} \mathrm{C}\right) ;{ }^{1} \mathrm{H}$ NMR $\left(300 \mathrm{MHz}, \mathrm{CDCl}_{3}\right): \delta 2.40(\mathrm{~s}, 3 \mathrm{H}), 7.18-7.66(\mathrm{~m}, 10 \mathrm{H}), 8.08-8.12(\mathrm{~m}, 1 \mathrm{H}), 8.66-8.74(\mathrm{~m}, 2 \mathrm{H}) ;{ }^{13} \mathrm{C} \mathrm{NMR}(75$ $\left.\mathrm{MHz}, \mathrm{CDCl}_{3}\right): \delta 17.31,122.31,122.85,125.07,125.60,126.19,126.33,126.80,127.02,127.42,128.39,129.33$, 129.936, 130.35, 131.88, 132.30, 137.06, 140.70; HRMS calcd for $\mathrm{C}_{21} \mathrm{H}_{16}$ : 268.1252, found: 268.1241 .

9-Butylphenanthrene (2d): This compound was prepared from 2,2'-dibromobiphenyl and 1-hexyne. GC yield: 75\%, Isolated yield: $58 \%$, colorless solid $\mathrm{mp}=78-79{ }^{\circ} \mathrm{C}\left(\right.$ lit. $\left.^{7} 79.5-80.5{ }^{\circ} \mathrm{C}\right) ;{ }^{1} \mathrm{H} \mathrm{NMR}\left(300 \mathrm{MHz}, \mathrm{CDCl}_{3}\right): 80.95(\mathrm{t}, J=7$ $\mathrm{Hz}, 3 \mathrm{H}), 1.43$ (qt, $J=7,7 \mathrm{~Hz}, 2 \mathrm{H}), 1.74$ (tt, $J=7,7 \mathrm{~Hz}, 2 \mathrm{H}), 3.03$ (t, $J=7 \mathrm{~Hz}, 2 \mathrm{H}), 7.50-7.56(\mathrm{~m}, 5 \mathrm{H}), 7.74(\mathrm{~m}, 1$ $\mathrm{H}), 8.04(\mathrm{~d}, J=7 \mathrm{~Hz}, 1 \mathrm{H}), 8.55(\mathrm{~d}, J=4 \mathrm{~Hz}, 1 \mathrm{H}), 8.63(\mathrm{~d}, J=4 \mathrm{~Hz}, 1 \mathrm{H}) ;{ }^{13} \mathrm{C} \mathrm{NMR}\left(75 \mathrm{MHz}, \mathrm{CDCl}_{3}\right): \delta 14.04$, $22.91,32.36,33.13,122.41,123.18,124.45,125.79,125.91,126.02,126.39,126.50,127.98,129.62,130.72,131.36$, 131.98, 136.90; HRMS calcd for $\mathrm{C}_{18} \mathrm{H}_{18}$ : 234.1409, found: 234.1394 .

9-Phenylphenanthrene (2e): This compound was prepared from 2,2'-dibromobiphenyl and phenylacetylene. GC yield: $63 \%$, Isolated yield: $45 \%$, colorless solid, $\mathrm{mp}=86-87{ }^{\circ} \mathrm{C} ;{ }^{1} \mathrm{H}$ NMR $\left(300 \mathrm{MHz}, \mathrm{CDCl}_{3}\right): \delta 7.28-7.55(\mathrm{~m}, 10 \mathrm{H})$, $7.73-7.82(\mathrm{~m}, 2 \mathrm{H}), 8.55(\mathrm{dd}, J=8.08 \mathrm{~Hz}, 1 \mathrm{H}), 8.61(\mathrm{~d}, J=8.26 \mathrm{~Hz}, 2 \mathrm{H}) ;{ }^{13} \mathrm{C} \mathrm{NMR}\left(75 \mathrm{MHz}, \mathrm{CDCl}_{3}\right): \delta 122.50$, $122.87,126.41,126.47,126.54,126.80,126.89,127.32,127.49,128.27,128.63,129.93,130.04,130.60,131.10$, 131.53, 138.74, 140.77; HRMS calcd for $\mathrm{C}_{20} \mathrm{H}_{14}$ : 254.1096, found: 254.1098 .

9-(1-Butynyl)-10-ethylphenanthrene (2f): This compound was prepared from 2,2'-dibromobiphenyl and 3,5-octadiyne. GC yield: $67 \%$, Isolated yield: $47 \%$, colorless solid, mp $=92-93{ }^{\circ} \mathrm{C}$; ${ }^{1} \mathrm{H} \mathrm{NMR}\left(300 \mathrm{MHz}, \mathrm{CDCl}_{3}\right)$ : $\delta 1.38(\mathrm{t}, J=7.6 \mathrm{~Hz}, 3 \mathrm{H}), 1.41(\mathrm{t}, J=7.5 \mathrm{~Hz}, 3 \mathrm{H}), 2.66(\mathrm{q}, J=7.5 \mathrm{~Hz}, 2 \mathrm{H}), 4.43(\mathrm{q}, J=7.5 \mathrm{~Hz}, 2 \mathrm{H}), 7.58-7.67(\mathrm{~m}, 4$

\footnotetext{
Gilman, H; Gaj, B. J. J. Org. Chem. 1957, 22, 447-449.

Hart, H.; Harada, K.; Du, C.-J. F. J. Org. Chem. 1985, 50, 3104

Campo, M. A.; Larock, R. C. Org. Lett. 2000, 2, 3675.

Inhoffen, H. H.; Kampe, D.; Cordes, C.; Müller, K.-D.; Milkowski, W. Liebigs Ann. Chem. 1964, 674, 36.

Ohwada, T.; Shudo, K. J. Am. Chem. Soc. 1988, 110, 1862.

Bradsher, C. K.; Rosher, R. J. Am. Chem. Soc. 1939, 61, 1524

Bradsher, C. K.; Amore, S. T. J. Am. Chem. Soc. 1941, 63, 493.
} 
$\mathrm{H}), 8.10-8.14(\mathrm{~m}, 1 \mathrm{H}), 8.48-8.51(\mathrm{~m}, 1 \mathrm{H}), 8.63-8.72(\mathrm{~m}, 2 \mathrm{H}) ;{ }^{13} \mathrm{C}$ NMR $\left(75 \mathrm{MHz}, \mathrm{CDCl}_{3}\right): \delta 13.65,14.27,14.39$, $24.88,77.17,100.59,118.55,122.36,123.09,124.88,126.11,126.48,126.73,126.83,127.01,129.12,130.11,130.33$, 131.52, 142.18; HRMS calcd for $\mathrm{C}_{20} \mathrm{H}_{18}$ : 258.1409, found: 258.1421.

9-Phenyl-10-phenylethynylphenanthrene (2g): This compound was prepared from 2,2'-dibromobiphenyl and 1,4-diphenyl-1,3-butadiyne. Isolated yield: $45 \%$, colorless solid, $\mathrm{mp}=146-147{ }^{\circ} \mathrm{C}\left(\right.$ lit. $\left.{ }^{8} 143-144{ }^{\circ} \mathrm{C}\right) ;{ }^{1} \mathrm{H} \mathrm{NMR}(300$ $\left.\mathrm{MHz}, \mathrm{CDCl}_{3}\right): \delta 7.19-7.24(\mathrm{~m}, 5 \mathrm{H}), 7.44-7.71(\mathrm{~m}, 10 \mathrm{H}), 8.62-8.72(\mathrm{~m}, 3 \mathrm{H}) ;{ }^{13} \mathrm{C}$ NMR $\left(75 \mathrm{MHz}, \mathrm{CDCl}_{3}\right): \delta 87.76$, $98.21,119.03,122.60,122.63,123.47,126.72,127.02,127.14,127.27,127.34,127.46,127.74,128.02,128.12,128.21$, 129.68, 130.19, 130.66 (2C), 131.33, 131.40, 139.76, 142.94; HRMS calcd for $\mathrm{C}_{28} \mathrm{H}_{18}$ : 354.1409, found: 354.1404 .

9-Phenyl-10-trimethylsilylphenanthrene (2h): This compound was prepared from 2,2'-dibromobiphenyl and 1-phenyl-2-trimethylsilylacetylene, and purified by Kugel-rohr distillation followed by recrystallization from pentane. GC yield: $88 \%$, Isolated yield: $75 \%$, colorless solid, $\mathrm{mp}=172-173{ }^{\circ} \mathrm{C} ;{ }^{1} \mathrm{H} \mathrm{NMR}\left(300 \mathrm{MHz}, \mathrm{CDCl}_{3}\right): \delta 0.12(\mathrm{~s}, 9 \mathrm{H})$, 7.37-7.50 (m, $3 \mathrm{H}), 7.60-7.69(\mathrm{~m}, 7 \mathrm{H}), 8.33(\mathrm{~d}, J=7.7 \mathrm{~Hz}, 1 \mathrm{H}), 8.73-8.81(\mathrm{~d}, 2 \mathrm{H})$; HRMS calcd for $\mathrm{C}_{23} \mathrm{H}_{22} \mathrm{Si}$ : 326.1491, found: 326.1502 .

9-Ethoxycarbonyl-10-methylphenanthrene (2i): This compound was prepared from 2,2'-dibromobiphenyl and ethyl 2-butynoate. GC yield: $46 \%$, Isolated yield: $31 \%$, colorless solid, $\mathrm{mp}=88-89{ }^{\circ} \mathrm{C}$ (lit. $\left.{ }^{9} 74{ }^{\circ} \mathrm{C}\right)$; ${ }^{1} \mathrm{H} \mathrm{NMR}(300 \mathrm{MHz}$, $\left.\mathrm{CDCl}_{3}\right): \delta 1.40(\mathrm{t}, J=7.2 \mathrm{~Hz}, 3 \mathrm{H}$ ), $2.62(\mathrm{~s}, 3 \mathrm{H}), 4.47$ (q, $J=7.1 \mathrm{~Hz}, 2 \mathrm{H}), 7.48-7.69(\mathrm{~m}, 5 \mathrm{H}), 8.02-8.05(\mathrm{dd}, 1 \mathrm{H})$, 8.58-8.61 (d, $1 \mathrm{H}), 8.63(\mathrm{~d}, J=2.2 \mathrm{~Hz}, 1 \mathrm{H}) ;{ }^{13} \mathrm{C} \mathrm{NMR}\left(75 \mathrm{MHz}, \mathrm{CDCl}_{3}\right): \delta 14.36,17.06,61.47,122.70,122.94$, $125.08,125.17,126.39,127.00,127.12,127.23,128.13,129.40,129.56,130.37,130.39,130.95,170.35$; HRMS calcd for $\mathrm{C}_{18} \mathrm{H}_{16} \mathrm{O}_{2}$ : 264.1150, found: 264.1132 .

9,10-Bis(methoxycarbonyl)phenanthrene (2j): This compound was prepared from 2,2'-dibromobiphenyl and dimethyl acetylenedicarboxylate. $\mathrm{GC}$ yield: $54 \%$, Isolated yield: $43 \%$, colorless solid, $\mathrm{mp}=129-130{ }^{\circ} \mathrm{C}\left(\right.$ lit. ${ }^{10}$ $\left.130{ }^{\circ} \mathrm{C}\right) ;{ }^{1} \mathrm{H}$ NMR $\left(300 \mathrm{MHz}, \mathrm{CDCl}_{3}\right): \delta 4.02(\mathrm{~s}, 6 \mathrm{H}), 7.60-7.71(\mathrm{~m}, 4 \mathrm{H}), 8.12(\mathrm{~d}, J=8.1 \mathrm{~Hz}, 2 \mathrm{H}), 8.63(\mathrm{~d}, J=8.1 \mathrm{~Hz}$, $2 \mathrm{H}) ;{ }^{13} \mathrm{C}$ NMR $\left(75 \mathrm{MHz}, \mathrm{CDCl}_{3}\right): \delta 52.73,122.77,126.75,126.91,127.55,128.38,129.77,130.93$, 168.28; HRMS calcd for $\mathrm{C}_{18} \mathrm{H}_{14} \mathrm{O}_{4} 294.0892$, found 294.0871 .

Synthesis of 1-Butyl-2,3,4-triphenylnaphthalene (3): Trans-1-lithio-2-o-lithiophenyl-1-phenyl-1-hexene was prepared from diphenylacetylene $(178 \mathrm{mg}, 1.0 \mathrm{mmol})$ and $n$-butyllithium $(1.28 \mathrm{~mL}, 1.56 \mathrm{M}, 2.0 \mathrm{mmol})$ in hexane and TMEDA according to the literatures. ${ }^{11}$ The mixture was added with chromium(III) chloride (158 $\left.\mathrm{mg}, 1.0 \mathrm{mmol}\right) \mathrm{and}$ stirred at room temperature for $1 \mathrm{~h}$. After addition of diphenylacetylene (356 mg, $2.0 \mathrm{mmol})$, the mixture was heated to $50{ }^{\circ} \mathrm{C}$ for $6 \mathrm{~h}$. The mixture was quenched by addition of $3 \mathrm{~N} \mathrm{HCl}$ solution and extracted with ethyl acetate. The combined organic layer was dried with magnesium sulfate, and concentrated in vacuo. The residue was purified by a flash column chromatography on silica gel (hexane as eluent) to afford 1-butyl-2,3,4-triphenylnaphthalene 3 . GC yield: $99 \%$, Isolated yield: $87 \%$, colorless solid, ${ }^{1} \mathrm{H}$ NMR $\left(300 \mathrm{MHz}, \mathrm{CDCl}_{3}\right): 80.80(\mathrm{t}, J=7.3 \mathrm{~Hz}, 3 \mathrm{H}), 1.25-1.36(\mathrm{~m}$, $2 \mathrm{H}), 1.60-1.71(\mathrm{~m}, 2 \mathrm{H}), 2.91(\mathrm{t}, J=8.3 \mathrm{~Hz}, 2 \mathrm{H}), 6.74-6.84(\mathrm{~m}, 5 \mathrm{H}), 7.04-7.23(\mathrm{~m}, 10 \mathrm{H}), 7.34-7.39(\mathrm{~m}, 1 \mathrm{H})$, $7.51-7.55(\mathrm{~m}, 1 \mathrm{H}), 7.59(\mathrm{~d}, J=8.3 \mathrm{~Hz}, 1 \mathrm{H}), 8.16(\mathrm{~d}, J=8.4 \mathrm{~Hz}, 1 \mathrm{H}) ;{ }^{13} \mathrm{C} \mathrm{NMR}\left(75 \mathrm{MHz}, \mathrm{CDCl}_{3}\right): \delta 13.71,23.17$, $30.05,33.42,124.51,125.13,125.52,125.82,125.97,126.19,126.40,127.18,127.037,127.71,130.37,131.07,131.15$, 131.30, 132.33, 136.32, 136.83, 139.06, 139.08, 139.70, 140.80, 141.15; HRMS calcd for $\mathrm{C}_{32} \mathrm{H}_{28}$ : 412.2191, found: 412.2197.

General procedure for the synthesis of phenanthrene derivatives from monohalogenated biaryls: To a THF solution of monohalobiphenyl $(1.0 \mathrm{mmol}), n$-butyllithium $(1.28 \mathrm{~mL}, 1.56 \mathrm{M}, 2.0 \mathrm{mmol})$ was added at $-78{ }^{\circ} \mathrm{C}$ and stirred for $1 \mathrm{~h}$. Then the mixture was added with chromium(III) chloride (158 $\mathrm{mg}, 1.0 \mathrm{mmol})$ and warmed to room temperature. After $1 \mathrm{~h}$, an alkyne $(2 \mathrm{mmol})$ was added and the mixture was heated to $50{ }^{\circ} \mathrm{C}$. The mixture was quenched by addition of $3 \mathrm{~N} \mathrm{HCl}$ solution and extracted with ethyl acetate. The organic layer was separated, dried with magnesium sulfate, and concentrated in vacuo. The residue was purified by column chromatography on silica gel which afforded the corresponding phenanthrene.

9,10-Diethylphenanthrene (2a): This compound was obtained from 2-bromobiphenyl and 3-hexyne in 69\% GC yield. The spectral data were shown above.

\footnotetext{
8 Yao, T.; Campo, M. A.; Larock, R. C. Org. Lett. 2004, 6, 2677.

9 Peña, D.; Pérez, D.; Guitián, E.; Castedo, L. J. Am. Chem. Soc. 1999, 121, 5827.

10 Peña, D.; Pérez, D.; Guitián, E.; Castedo, L. J. Org. Chem. 2000, 65, 6944.

(a) Rausch, M. D.; Klemann, L. P. J. Am. Chem. Soc. 1967, 89, 5732. (b) Mulvaney, J. E; Garlund, Z. G; Garlund, S. L; Newton, D. J. J. Am. Chem. Soc. 1966, 88, 476.
} 
2-Methyl-9,10-diethylphenanthrene (5a): This compound was prepared from 2-iodo-4'-methylbiphenyl and 3-hexyne. GC yield: $68 \%$, Isolated yield: $58 \%$, colorless solid, mp $=109-110{ }^{\circ} \mathrm{C} ;{ }^{1} \mathrm{H} \mathrm{NMR}\left(300 \mathrm{MHz}, \mathrm{CDCl}_{3}\right): \delta 1.29$ $(\mathrm{t}, J=7.5 \mathrm{~Hz}, 6 \mathrm{H}), 2.52(\mathrm{~s}, 3 \mathrm{H}), 3.08-3.15(\mathrm{~m}, 4 \mathrm{H}), 7.36(\mathrm{~d}, J=8.4 \mathrm{~Hz}, 1 \mathrm{H}), 7.47-7.53(\mathrm{~m}, 2 \mathrm{H}), 7.82(\mathrm{~s}, 1 \mathrm{H})$, 8.02-8.05 (m, $1 \mathrm{H}), 8.51(\mathrm{~d}, J=8.4 \mathrm{~Hz}, 1 \mathrm{H}), 8.59-8.62(\mathrm{~m}, 1 \mathrm{H}) ;{ }^{13} \mathrm{C}$ NMR $\left(75 \mathrm{MHz}, \mathrm{CDCl}_{3}\right): \delta 15.03(2 \mathrm{C}), 21.99$, $22.02,22.09,122.75,122.89,124.24,124.52,125.28,126.12,127.06,127.67,129.89,130.66,131.16,134.52,134.81$, 136.11; HRMS calcd for $\mathrm{C}_{19} \mathrm{H}_{20}: 248.1565$, found: 248.1572 .

5,6-Diethylbenzo[c]phenanthrene (7a): This compound was prepared from 1-(2-iodophenyl)naphthalene and 3-hexyne. Isolated yield: $58 \%$, colorless solid, ${ }^{1} \mathrm{H}$ NMR $\left(300 \mathrm{MHz}, \mathrm{CDCl}_{3}\right): \delta 1.35(\mathrm{t}, J=8.1 \mathrm{~Hz}, 3 \mathrm{H}), 1.78(\mathrm{t}, J=$ $8.1 \mathrm{~Hz}, 3 \mathrm{H}), 3.18-3.27$ (m, $4 \mathrm{H}), 7.51-7.61(\mathrm{~m}, 4 \mathrm{H}), 7.87(\mathrm{~d}, J=9.0 \mathrm{~Hz}, 1 \mathrm{H}), 7.93-7.95(\mathrm{~m}, 1 \mathrm{H}), 8.05(\mathrm{~d}, J=9.0 \mathrm{~Hz}$, $1 \mathrm{H}), 8.19(\mathrm{~d}, J=7.9 \mathrm{~Hz}, 1 \mathrm{H}), 8.96(\mathrm{dd}, J=7.3 \mathrm{~Hz}, J=7.5 \mathrm{~Hz}, 2 \mathrm{H}) ;{ }^{13} \mathrm{C} \mathrm{NMR}\left(75 \mathrm{MHz}, \mathrm{CDCl}_{3}\right): \delta 15.20,15.48$, $22.13,22.32,122.57,123.99,124.45,125.49,125.65,125.88,127.03,127.23,127.85,128.63,128.87,129.42,129.57$, 130.224, 131.98, 132.60, 134.91, 135.33; HRMS calcd for $\mathrm{C}_{22} \mathrm{H}_{20}: 284.1565$, found: 284.1590.

5,6-Diphenylbenzo[c]phenanthrene (7b): This compound was prepared from 1-(2-iodophenyl)naphthalene and diphenylacetylene. Isolated yield: $28 \%$, colorless solid, ${ }^{1} \mathrm{H}$ NMR $\left(300 \mathrm{MHz}, \mathrm{CDCl}_{3}\right): \delta 7.07-7.17(\mathrm{~m}, 10 \mathrm{H})$, 7.40-7.68 (m, $7 \mathrm{H}), 7.88(\mathrm{~d}, J=7.7 \mathrm{~Hz}, 1 \mathrm{H}), 9.05(\mathrm{~d}, J=8.3 \mathrm{~Hz}, 2 \mathrm{H}) ;{ }^{13} \mathrm{C} \mathrm{NMR}\left(75 \mathrm{MHz}, \mathrm{CDCl}_{3}\right): \delta 125.26,125.60$, $125.98,126.01,126.05,126.50,126.52,127.06,127.37,127.44,127.56,127.59,128.14,128.23,128.52,129.70$, $129.85,130.11,131.07,131.18,132.74,133.10,137.16,137.51,139.45,139.63$; HRMS calcd for $\mathrm{C}_{30} \mathrm{H}_{20}: 380.1565$, found: 380.1572 .

9,10-Diethylbenzo[g]chrysene (9): 9-Phenyl-10-trimethylsilylphenanthrene $\quad \mathbf{2 h}$ was converted into 9-iodo-10-phenylphenanthrene $\mathbf{8}$ by the treatment with iodine monochloride according to the literature. ${ }^{3}$ Dilithiation of iodophenanthrene $8(380 \mathrm{mg}, 1.0 \mathrm{mmol})$ was carried out in toluene with $n$-butyllithium $(1.28 \mathrm{~mL}, 1.56 \mathrm{M}, 2.0$ $\mathrm{mmol})$ at $0{ }^{\circ} \mathrm{C}$ for $1 \mathrm{~h}$. The resulting mixture was added with chromium(III) chloride (158 $\left.\mathrm{mg}, 1.0 \mathrm{mmol}\right)$ and THF, and stirred at room temperature for $1 \mathrm{~h}$. After addition of 3-hexyne $(0.23 \mathrm{~mL}, 2.0 \mathrm{mmol})$, the mixture was heated to $50{ }^{\circ} \mathrm{C}$ for $12 \mathrm{~h}$. The reaction was quenched by addition of $3 \mathrm{~N} \mathrm{HCl}$ solution and extracted with ethyl acetate. The organic layer was dried with magnesium sulfate, and concentrated in vacuo. The residue was purified by a flash column chromatography on silica gel (hexane as eluent) to afford the corresponding benzo $[g]$ chrysene 9. Isolated yield: $22 \%$, colorless solid, ${ }^{1} \mathrm{H}$ NMR $\left(300 \mathrm{MHz} \mathrm{CDCl}_{3}\right): \delta 1.39(\mathrm{t}, J=7.4 \mathrm{~Hz}, 3 \mathrm{H}), 1.45(\mathrm{t}, J=7.3 \mathrm{~Hz}, 3 \mathrm{H}), 3.35(\mathrm{q}, J$ $=8.1 \mathrm{~Hz}, 2 \mathrm{H}), 3.40(\mathrm{q}, J=7.4 \mathrm{~Hz}, 2 \mathrm{H}), 7.49-7.61(\mathrm{~m}, 6 \mathrm{H}), 8.20(\mathrm{~d}, J=8.1 \mathrm{~Hz}, 1 \mathrm{H}), 8.29(\mathrm{~d}, J=7.9 \mathrm{~Hz}, 1 \mathrm{H})$, 8.60-8.71 (m, $4 \mathrm{H}) ;{ }^{13} \mathrm{C}$ NMR $\left(75 \mathrm{MHz}, \mathrm{CDCl}_{3}\right): \delta 15.45,16.87,21.86,25.49,123.40,123.50,123.95,124.66,125.68$, $125.85,126.12,126.14,126.46,128.28,128.40,128.60,128.65,129.33,129.74,129.80,130.37,130.54(2 \mathrm{C}), 131.76$, 134.76, 137.35; HRMS calcd for $\mathrm{C}_{26} \mathrm{H}_{22}$ : 334.1721, found: 334.1740 .

General procedure for the synthesis of pyrrolo[1,2-a]quinoline derivatives 11: To a hexane solution of 1-phenylpyrrole $10(143 \mathrm{mg}, 1.0 \mathrm{mmol})$ was added $n$-butyllithium $(1.28 \mathrm{~mL}, 1.56 \mathrm{M}, 2.0 \mathrm{mmol})$ in the presence of TMEDA $(0.30 \mathrm{~mL}, 2.0 \mathrm{mmol})$ at $-78^{\circ} \mathrm{C}$. ${ }^{12}$ Then, the mixture was added chromium(III) chloride $(158 \mathrm{mg}, 1.0 \mathrm{mmol})$ and THF and stirred at room temperature. After $1 \mathrm{~h}$, an alkyne $(2 \mathrm{mmol})$ was added and the mixture was heated to $50{ }^{\circ} \mathrm{C}$ for $12 \mathrm{~h}$. The reaction was quenched by addition of $3 \mathrm{~N} \mathrm{HCl}$ solution and extracted with ethyl acetate. The organic layer was dried over magnesium sulfate, and concentrated in vacuo. The residue was purified by a flash column chromatography on silica gel (hexane as eluent) to afford the corresponding pyrrolo[1,2-a]quinoline derivatives 11.

4,5-Diethyl-pyrrolo[1,2-a]quinoline (11a): This compound was prepared from 1-phenylpyrrole and 3-hexyne. GC yield: $73 \%$, Isolated yield: $68 \%$, colorless solid, $\mathrm{mp}=68-69{ }^{\circ} \mathrm{C} ;{ }^{1} \mathrm{H}$ NMR $\left(300 \mathrm{MHz}, \mathrm{CDCl}_{3}\right): \delta 1.19(\mathrm{t}, J=7.4 \mathrm{~Hz}, 3$ $\mathrm{H}), 1.23(\mathrm{t}, J=7.5 \mathrm{~Hz}, 3 \mathrm{H}), 2.78(\mathrm{q}, J=7.5 \mathrm{~Hz}, 2 \mathrm{H}), 2.86(\mathrm{q}, J=7.5 \mathrm{~Hz}, 2 \mathrm{H}), 6.42(\mathrm{~d}, J=2.6 \mathrm{~Hz}, 1 \mathrm{H}), 6.68(\mathrm{t}, J=$ $3.2 \mathrm{~Hz}, 1 \mathrm{H}), 7.23-7.39(\mathrm{~m}, 2 \mathrm{H}), 7.71-7.84(\mathrm{~m}, 3 \mathrm{H}) ;{ }^{13} \mathrm{C}$ NMR $\left(75 \mathrm{MHz}, \mathrm{CDCl}_{3}\right): \delta 14.23,14.92,20.35,22.52,100.46$, $111.47,112.31,114.37,123.32,123.76,125.04,126.22,126.50,129.88,131.79,132.83$; HRMS calcd for $\mathrm{C}_{16} \mathrm{H}_{17} \mathrm{~N}$ : 223.1361, found: 223.1364 .

4,5-Diphenyl-pyrrolo[1,2-a]quinoline (11b): This compound was prepared from 1-phenylpyrrole and diphenylacetylene. $\mathrm{GC}$ yield: $99 \%$, Isolated yield: $83 \%$, colorless solid, $\mathrm{mp}=210-211{ }^{\circ} \mathrm{C}$; ${ }^{1} \mathrm{H} \mathrm{NMR}(300 \mathrm{MHz}$, $\left.\mathrm{CDCl}_{3}\right): \delta 6.24-6.25(\mathrm{~m}, 1 \mathrm{H}), 6.77-6.80(\mathrm{dd}, J=3.3 \mathrm{~Hz}, 1 \mathrm{H}), 7.15-7.29(\mathrm{~m}, 11 \mathrm{H}), 7.41-7.44(\mathrm{~m}, 1 \mathrm{H}), 7.49-7.55(\mathrm{~m}, 1$

2 Faigl, F.; Schlosser, M. Tetrahedron 1993, 49, 10271. 
H) , $7.96(\mathrm{~d}, J=8.1 \mathrm{~Hz}, 2 \mathrm{H}) ;{ }^{13} \mathrm{C}$ NMR $\left(75 \mathrm{MHz}, \mathrm{CDCl}_{3}\right): \delta 104.27,112.26,112.91,114.04,123.38,124.74,126.71$, 126.93, 127.56, 127.69, 127.76, 128.11, 128.41, 130.20, 130.83, 131.47, 131.99, 132.71, 137.60, 137.72; HRMS calcd for $\mathrm{C}_{24} \mathrm{H}_{17} \mathrm{~N}$ : 319.1361 , found: 319.1361 .

Synthesis of 9b-azacyclopenta[cd] phenalene derivatives 12: 4,5-Diethyl-pyrrolo[1,2- $a$ ]quinoline 11a (234 mg, 1.0 mmol) was dilithiated by the addition of $n$-butyllithium $(1.28 \mathrm{~mL}, 1.56 \mathrm{M}, 2.0 \mathrm{mmol})$ at $-78^{\circ} \mathrm{C}$ for $1 \mathrm{~h}$ in hexane in the presence of TMEDA $(0.302 \mathrm{~mL}, 2.0 \mathrm{mmol})$. Then, to a solution of the reaction was added chromium(III) chloride $(0.158 \mathrm{~g}, 1.0 \mathrm{mmol})$ and THF at room temperature. After $1 \mathrm{~h}$, an alkyne $(2 \mathrm{mmol})$ was added and the mixture was heated to $50{ }^{\circ} \mathrm{C}$. The mixture was quenched by addition of $3 \mathrm{~N} \mathrm{HCl}$ solution and extracted with ethyl acetate. The organic layer was dried over magnesium sulfate, and concentrated in vacuo. The residue was purified by a flash column chromatography on silica gel (hexane as eluent) to afford the corresponding $9 \mathrm{~b}$-azacyclopenta[cd]phenalene derivatives 12.

3,4,8,9-Tetraethyl-9b-azacyclopenta[cd]phenalene (12a): This compound was prepared from pyrroloquinoline 11a and 3-hexyne. Isolated yield: $39 \%$, Yellow solid, $\mathrm{mp}=193-194{ }^{\circ} \mathrm{C} ;{ }^{1} \mathrm{H}$ NMR $\left(400 \mathrm{MHz}\right.$, Benzene- $\left.d_{6}\right): \delta 1.18(\mathrm{t}, J=$ $7.5 \mathrm{~Hz}, 6 \mathrm{H}), 1.26(\mathrm{t}, J=7.6 \mathrm{~Hz}, 6 \mathrm{H}), 2.73-2.84(\mathrm{~m}, 8 \mathrm{H}), 6.97(\mathrm{~s}, 2 \mathrm{H}), 7.47$ (s, $3 \mathrm{H}) ;{ }^{13} \mathrm{C}$ NMR $(100 \mathrm{MHz}$, Benzene- $\left.d_{6}\right): \delta 13.94,15.52,21.65,23.30,104.07,116.72,124.09,126.19,127.79,128.05,130.78,132.65$; HRMS calcd for $\mathrm{C}_{22} \mathrm{H}_{25} \mathrm{~N}$ : 303.1987 , found: 303.1984 .

3,4-Diethyl-8,9-diphenyl-9b-azacyclopenta[cd $]$ phenalene (12b): This compound was prepared from pyrroloquinoline 11a and diphenylacetylene. Isolated yield: $55 \%$, Light yellow solid, $\mathrm{mp}=225-226{ }^{\circ} \mathrm{C} ;{ }^{1} \mathrm{H} \mathrm{NMR}(00$ MHz, Benzene- $\left.d_{6}\right): \delta 1.30$ (t, $\left.J=7.5 \mathrm{~Hz}, 3 \mathrm{H}\right), 1.38$ (t, $\left.J=7.5 \mathrm{~Hz}, 3 \mathrm{H}\right), 2.84-2.94(\mathrm{~m}, 4 \mathrm{H}), 6.98-7.01$ (m, $2 \mathrm{H}$ ), 7.09-7.46 (m, $12 \mathrm{H}), 7.53(\mathrm{~d}, J=7.7 \mathrm{~Hz}, 1 \mathrm{H}) ;{ }^{13} \mathrm{C}$ NMR (100 MHz, Benzene- $\left.d_{6}\right): \delta 13.86,15.50,21.68,23.30,104.78$, $107.31,117.48,119.46,124.14,125.95,127.53,127.68,127.91,128.02,128.32,128.78,129.31,130.02,131.08$, 131.30, 131.86, 132.02, 133.14, 138.91, 139.24; HRMS calcd for $\mathrm{C}_{30} \mathrm{H}_{25} \mathrm{~N}$ : 399.1987, found: 399.1964 .

3,4-Diethyl-8,9-di-p-tolyl-9b-azacyclopenta[cd]phenalene (12c): This compound was prepared from pyrroloquinoline 11a and di- $p$-tolylacetylene. Isolated yield: $49 \%$, Light yellow solid, mp $=196-197{ }^{\circ} \mathrm{C} ;{ }^{1} \mathrm{H} \mathrm{NMR}$ $\left(300 \mathrm{MHz}\right.$, Benzene- $\left.d_{6}\right): \delta 1.21(\mathrm{t}, J=7.4 \mathrm{~Hz}, 3 \mathrm{H}), 1.28(\mathrm{t}, J=7.5 \mathrm{~Hz}, 3 \mathrm{H}), 2.01(\mathrm{~s}, 3 \mathrm{H}), 2.05(\mathrm{~s}, 3 \mathrm{H}), 2.74-2.85(\mathrm{~m}$, $4 \mathrm{H}), 6.93-6.99(\mathrm{~m}, 5 \mathrm{H}), 7.17-7.98(\mathrm{~m}, 5 \mathrm{H}), 7.29-7.35(\mathrm{~m}, 2 \mathrm{H}), 7.42-7.47(\mathrm{~m}, 1 \mathrm{H}) ;{ }^{13} \mathrm{C}$ NMR $\left(75 \mathrm{MHz}, \mathrm{DMSO}-d_{6}\right.$, $423 \mathrm{~K}): \delta 11.90,13.36,19.37,19.69,19.71,20.93,103.14,105.14,115.86,117.36,122.51,123.85,125.52,126.24$, 126.36, 127.34, 127.51, 128.07, 128.11, 129.03, 129.59, 129.71, 129.88, 130.51, 133.61, 133.95, 135.21, 135.46; HRMS calcd for $\mathrm{C}_{32} \mathrm{H}_{29} \mathrm{~N}$ : 427.2300 , found: 427.2293 .

3,4-Diethyl-8,9-di(2-thienyl)-9b-azacyclopenta[cd]phenalene (12d): This compound was prepared from pyrroloquinoline 11a and bis(2-thienyl)acetylene. Isolated yield: $38 \%$, Light yellow solid, $\mathrm{mp}=188-189{ }^{\circ} \mathrm{C} ;{ }^{1} \mathrm{H}$ NMR (300 MHz, Benzene- $\left.d_{6}\right): \delta 1.17(\mathrm{t}, J=7.8 \mathrm{~Hz}, 3 \mathrm{H}), 1.25(\mathrm{t}, J=7.5 \mathrm{~Hz}, 3 \mathrm{H}), 2.68-2.79(\mathrm{~m}, 4 \mathrm{H}), 6.74-4.77$ (m, 1 H), 6.82-6.93 (m, $3 \mathrm{H}), 6.98(\mathrm{~d}, J=4.6 \mathrm{~Hz}, 1 \mathrm{H}), 7.13-7.15(\mathrm{~m}, 2 \mathrm{H}), 7.27(\mathrm{dd}, J=7.9 \mathrm{~Hz}, 1 \mathrm{H}), 7.36$ (d, $J=7.7 \mathrm{~Hz}, 1$ H), $7.47(\mathrm{~d}, J=7.7 \mathrm{~Hz}, 1 \mathrm{H}) ;{ }^{13} \mathrm{C}$ NMR $\left(75 \mathrm{MHz}\right.$, DMSO- $\left.d_{6}, 423 \mathrm{~K}\right): \delta 11.84,13.29,19.39,20.92,103.76,106.39$, $116.63,117.60,121.36,122.86,123.75,125.28,125.36,125.48,125.62,125.99,126.10,126.29,126.94,127.88$, $128.22,128.30,129.67,130.37,136.58,137.25$; HRMS calcd for $\mathrm{C}_{26} \mathrm{H}_{21} \mathrm{NS}: 411.1115$, found: 411.1118 . 
Table 1. Crystallographic Data and Experimental Details of 3,4-Diethyl-8,9-diphenyl-9b- azacyclopenta[cd]phenalene (12b)

\begin{tabular}{|c|c|}
\hline Compound & 9b-azacyclopenta $[c d]$ phenalene (12b) \\
\hline Formula & $\mathrm{C}_{30} \mathrm{H}_{25} \mathrm{~N}$ \\
\hline$M$ & 399.53 \\
\hline Crystal system & Monoclinic \\
\hline Space group & $P 2_{1} / n$ \\
\hline$a,(\AA)$ & $17.979(2)$ \\
\hline$b,(\AA)$ & $10.718(1)$ \\
\hline$c,(\AA)$ & $11.977(1)$ \\
\hline$\beta,\left(^{\circ}\right)$ & $104.943(8)$ \\
\hline$V,\left(\AA^{3}\right)$ & $2230.0(4)$ \\
\hline$Z$ & 4 \\
\hline$\mu($ Mo $K \alpha),\left(\mathrm{cm}^{-1}\right)$ & $4.854(4)$ \\
\hline Crystal color & yellow \\
\hline Crystal habit & prism \\
\hline Crystal size, $\left(\mathrm{mm}^{3}\right)$ & $0.20 \times 0.25 \times 0.30$ \\
\hline Dcalcd, $\left(\mathrm{g} / \mathrm{cm}^{3}\right)$ & 1.190 \\
\hline$F(000)$ & 848 \\
\hline Diffractometer & Rigaku AFC7S \\
\hline$\lambda(\operatorname{Mo~} K \alpha),(\AA)$ & 1.5418 \\
\hline $\mathrm{T},(\mathrm{K})$ & 298.2 \\
\hline Data Images & 44 exposures at 4.0 minutes per degree \\
\hline Oscillation Ranges $\left(\varphi=0.0^{\circ}, \chi=45.0^{\circ}\right)$ & $\omega 130.0-190.0^{\circ}$ with $5.0^{\circ}$ step \\
\hline Oscillation Ranges $\left(\varphi=180.0^{\circ}, \chi=45.0^{\circ}\right)$ & $\omega 0.0-162.0^{\circ}$ with $5.0^{\circ}$ step \\
\hline $2 \theta \max ,\left(^{\circ}\right)$ & 55 \\
\hline No. of reflections meads & 4215(Unique: 4215, Rint $=0.035)$ \\
\hline No. of reflections obsd $[\mathrm{I}>2 \sigma(\mathrm{I})]$ & 2852 \\
\hline No. of parameters refined & 281 \\
\hline$R$ & 0.0984 \\
\hline$R w$ & 0.1373 \\
\hline$S$, goodness of fit & 3.383 \\
\hline$(\Delta / \delta) \max$ & 0.0002 \\
\hline Largest diff peak, $\left(\mathrm{e} \AA^{-3}\right)$ & 0.59 \\
\hline Largest diff hole, $\left(\mathrm{e} \AA^{-3}\right)$ & -0.22 \\
\hline
\end{tabular}

$R=\Sigma|| F_{O}|-| F_{C}|| / \Sigma|F o|, R w=\left[\Sigma w(|F o|-|F c|)^{2} / \Sigma w\left|F_{O}\right|^{2}\right]^{1 / 2}, w=\left[\sigma c^{2}(F o)+\left(p^{2} / 4\right)|\mathrm{Fo}|^{2}\right]^{-1}, p=0.00063$ $S=\left[\Sigma w(|F o|-|F c|)^{2} /(m-n)\right]^{1 / 2}(m=$ no. of used reflections, $n=$ no. of refined parameters $)$

Table 2. Intramolecular Distances $(\AA)$ Involving the Nonhydrogen Atoms of 3,4-Diethyl-8,9-diphenyl-9b-azacyclopenta[cd]phenalene (12b)

\begin{tabular}{llll}
\hline $\mathrm{N}(1)-\mathrm{C}(1)$ & $1.373(5)$ & $\mathrm{C}(9)-\mathrm{C}(10)$ & $1.379(6)$ \\
$\mathrm{N}(1)-\mathrm{C}(9)$ & $1.385(5)$ & $\mathrm{C}(10)-\mathrm{C}(11)$ & $1.550(10)$ \\
$\mathrm{N}(1)-\mathrm{C}(12)$ & $1.378(5)$ & $\mathrm{C}(11)-\mathrm{C}(12)$ & $1.513(9)$ \\
$\mathrm{C}(1)-\mathrm{C}(2)$ & $1.398(5)$ & $\mathrm{C}(12)-\mathrm{C}(13)$ & $1.427(7)$ \\
$\mathrm{C}(1)-\mathrm{C}(6)$ & $1.397(5)$ & $\mathrm{C}(13)-\mathrm{C}(14)$ & $1.369(7)$ \\
$\mathrm{C}(2)-\mathrm{C}(3)$ & $1.360(7)$ & $\mathrm{C}(13)-\mathrm{C}(27)$ & $1.515(7)$ \\
$\mathrm{C}(2)-\mathrm{C}(14)$ & $1.451(7)$ & $\mathrm{C}(14)-\mathrm{C}(29)$ & $1.510(7)$ \\
$\mathrm{C}(3)-\mathrm{C}(4)$ & $1.222(9)$ & $\mathrm{C}(15)-\mathrm{C}(16)$ & $1.408(6)$ \\
$\mathrm{C}(4)-\mathrm{C}(5)$ & $1.318(8)$ & $\mathrm{C}(15)-\mathrm{C}(20)$ & $1.383(6)$ \\
$\mathrm{C}(5)-\mathrm{C}(6)$ & $1.375(6)$ & $\mathrm{C}(16)-\mathrm{C}(17)$ & $1.363(7)$ \\
$\mathrm{C}(6)-\mathrm{C}(7)$ & $1.464(5)$ & $\mathrm{C}(17)-\mathrm{C}(18)$ & $1.388(8)$ \\
$\mathrm{C}(7)-\mathrm{C}(8)$ & $1.365(5)$ & $\mathrm{C}(18)-\mathrm{C}(19)$ & $1.373(8)$ \\
$\mathrm{C}(7)-\mathrm{C}(15)$ & $1.491(5)$ & $\mathrm{C}(19)-\mathrm{C}(20)$ & $1.404(7)$ \\
$\mathrm{C}(8)-\mathrm{C}(9)$ & $1.442(6)$ & $\mathrm{C}(21)-\mathrm{C}(22)$ & $1.369(7)$ \\
$\mathrm{C}(8)-\mathrm{C}(21)$ & $1.507(6)$ & $\mathrm{C}(21)-\mathrm{C}(26)$ & $1.380(6)$
\end{tabular}



$\mathrm{C}(22)-\mathrm{C}(23)$
1.391(7)
C(25)-C(26)
1.373(6)
C(23)-C(24)
1.363(8)
C(27)-C(28)
$1.539(9)$
C(24)-C(25)
$1.371(8)$
C(29)-C(30)
1.53(1)

Distances are in angstroms. Estimated standard deviations in the least significant figure are given in parentheses.

Table 3. Intramolecular Bond Angles $\left(^{\circ}\right)$ Involving the Nonhydrogen Atoms of 3,4-Diethyl-8,9-diphenyl-9b-azacyclopenta[cd]phenalene (12b)

\begin{tabular}{llll}
\hline $\mathrm{C}(1)-\mathrm{N}(1)-\mathrm{C}(9)$ & $123.1(3)$ & $\mathrm{N}(1)-\mathrm{C}(12)-\mathrm{C}(13)$ & $120.2(4)$ \\
$\mathrm{C}(1)-\mathrm{N}(1)-\mathrm{C}(12)$ & $121.7(4)$ & $\mathrm{C}(11)-\mathrm{C}(12)-\mathrm{C}(13)$ & $136.4(5)$ \\
$\mathrm{C}(9)-\mathrm{N}(1)-\mathrm{C}(12)$ & $115.2(4)$ & $\mathrm{C}(12)-\mathrm{C}(13)-\mathrm{C}(14)$ & $119.2(4)$ \\
$\mathrm{N}(1)-\mathrm{C}(1)-\mathrm{C}(2)$ & $119.7(4)$ & $\mathrm{C}(12)-\mathrm{C}(13)-\mathrm{C}(27)$ & $116.2(5)$ \\
$\mathrm{N}(1)-\mathrm{C}(1)-\mathrm{C}(6)$ & $120.4(3)$ & $\mathrm{C}(14)-\mathrm{C}(13)-\mathrm{C}(27)$ & $124.6(5)$ \\
$\mathrm{C}(2)-\mathrm{C}(1)-\mathrm{C}(6)$ & $120.0(4)$ & $\mathrm{C}(2)-\mathrm{C}(14)-\mathrm{C}(13)$ & $120.0(4)$ \\
$\mathrm{C}(1)-\mathrm{C}(2)-\mathrm{C}(3)$ & $114.8(5)$ & $\mathrm{C}(2)-\mathrm{C}(14)-\mathrm{C}(29)$ & $117.1(6)$ \\
$\mathrm{C}(1)-\mathrm{C}(2)-\mathrm{C}(14)$ & $119.2(4)$ & $\mathrm{C}(13)-\mathrm{C}(14)-\mathrm{C}(29)$ & $122.9(6)$ \\
$\mathrm{C}(3)-\mathrm{C}(2)-\mathrm{C}(14)$ & $126.0(5)$ & $\mathrm{C}(7)-\mathrm{C}(15)-\mathrm{C}(16)$ & $119.9(4)$ \\
$\mathrm{C}(2)-\mathrm{C}(3)-\mathrm{C}(4)$ & $128.2(7)$ & $\mathrm{C}(7)-\mathrm{C}(15)-\mathrm{C}(20)$ & $121.2(4)$ \\
$\mathrm{C}(3)-\mathrm{C}(4)-\mathrm{C}(5)$ & $117.2(7)$ & $\mathrm{C}(16)-\mathrm{C}(15)-\mathrm{C}(20)$ & $118.8(4)$ \\
$\mathrm{C}(4)-\mathrm{C}(5)-\mathrm{C}(6)$ & $125.0(5)$ & $\mathrm{C}(15)-\mathrm{C}(16)-\mathrm{C}(17)$ & $120.9(5)$ \\
$\mathrm{C}(1)-\mathrm{C}(6)-\mathrm{C}(5)$ & $114.6(4)$ & $\mathrm{C}(16)-\mathrm{C}(17)-\mathrm{C}(18)$ & $120.4(5)$ \\
$\mathrm{C}(1)-\mathrm{C}(6)-\mathrm{C}(7)$ & $118.0(3)$ & $\mathrm{C}(17)-\mathrm{C}(18)-\mathrm{C}(19) 119.6(5)$ \\
$\mathrm{C}(5)-\mathrm{C}(6)-\mathrm{C}(7)$ & $127.4(4)$ & $\mathrm{C}(18)-\mathrm{C}(19)-\mathrm{C}(20)$ & $120.6(5)$ \\
$\mathrm{C}(6)-\mathrm{C}(7)-\mathrm{C}(8)$ & $120.2(4)$ & $\mathrm{C}(15)-\mathrm{C}(20)-\mathrm{C}(19) 119.7(5)$ \\
$\mathrm{C}(6)-\mathrm{C}(7)-\mathrm{C}(15)$ & $117.8(3)$ & $\mathrm{C}(8)-\mathrm{C}(21)-\mathrm{C}(22)$ & $120.9(4)$ \\
$\mathrm{C}(8)-\mathrm{C}(7)-\mathrm{C}(15)$ & $122.0(4)$ & $\mathrm{C}(8)-\mathrm{C}(21)-\mathrm{C}(26)$ & $119.5(4)$ \\
$\mathrm{C}(7)-\mathrm{C}(8)-\mathrm{C}(9)$ & $120.8(4)$ & $\mathrm{C}(22)-\mathrm{C}(21)-\mathrm{C}(26)$ & $119.6(4)$ \\
$\mathrm{C}(7)-\mathrm{C}(8)-\mathrm{C}(21)$ & $122.1(4)$ & $\mathrm{C}(21)-\mathrm{C}(22)-\mathrm{C}(23) 119.4(5)$ \\
$\mathrm{C}(9)-\mathrm{C}(8)-\mathrm{C}(21)$ & $117.2(3)$ & $\mathrm{C}(22)-\mathrm{C}(23)-\mathrm{C}(24) 121.0(6)$ \\
$\mathrm{N}(1)-\mathrm{C}(9)-\mathrm{C}(8)$ & $117.5(3)$ & $\mathrm{C}(23)-\mathrm{C}(24)-\mathrm{C}(25) 119.2(5)$ \\
$\mathrm{N}(1)-\mathrm{C}(9)-\mathrm{C}(10)$ & $110.2(4)$ & $\mathrm{C}(24)-\mathrm{C}(25)-\mathrm{C}(26) 120.5(5)$ \\
$\mathrm{C}(8)-\mathrm{C}(9)-\mathrm{C}(10)$ & $132.2(4)$ & $\mathrm{C}(21)-\mathrm{C}(26)-\mathrm{C}(25) 120.3(5)$ \\
$\mathrm{C}(9)-\mathrm{C}(10)-\mathrm{C}(11)$ & $105.1(4)$ & $\mathrm{C}(13)-\mathrm{C}(27)-\mathrm{C}(28) 112.0(5)$ \\
$\mathrm{C}(10)-\mathrm{C}(11)-\mathrm{C}(12)$ & $106.1(5)$ & $\mathrm{C}(14)-\mathrm{C}(29)-\mathrm{C}(30)$ & $111.3(6)$ \\
$\mathrm{N}(1)-\mathrm{C}(12)-\mathrm{C}(11)$ & $103.4(5)$ & &
\end{tabular}

Angles are in degrees. Estimated standard deviations in the least significant figure are given in parentheses. 

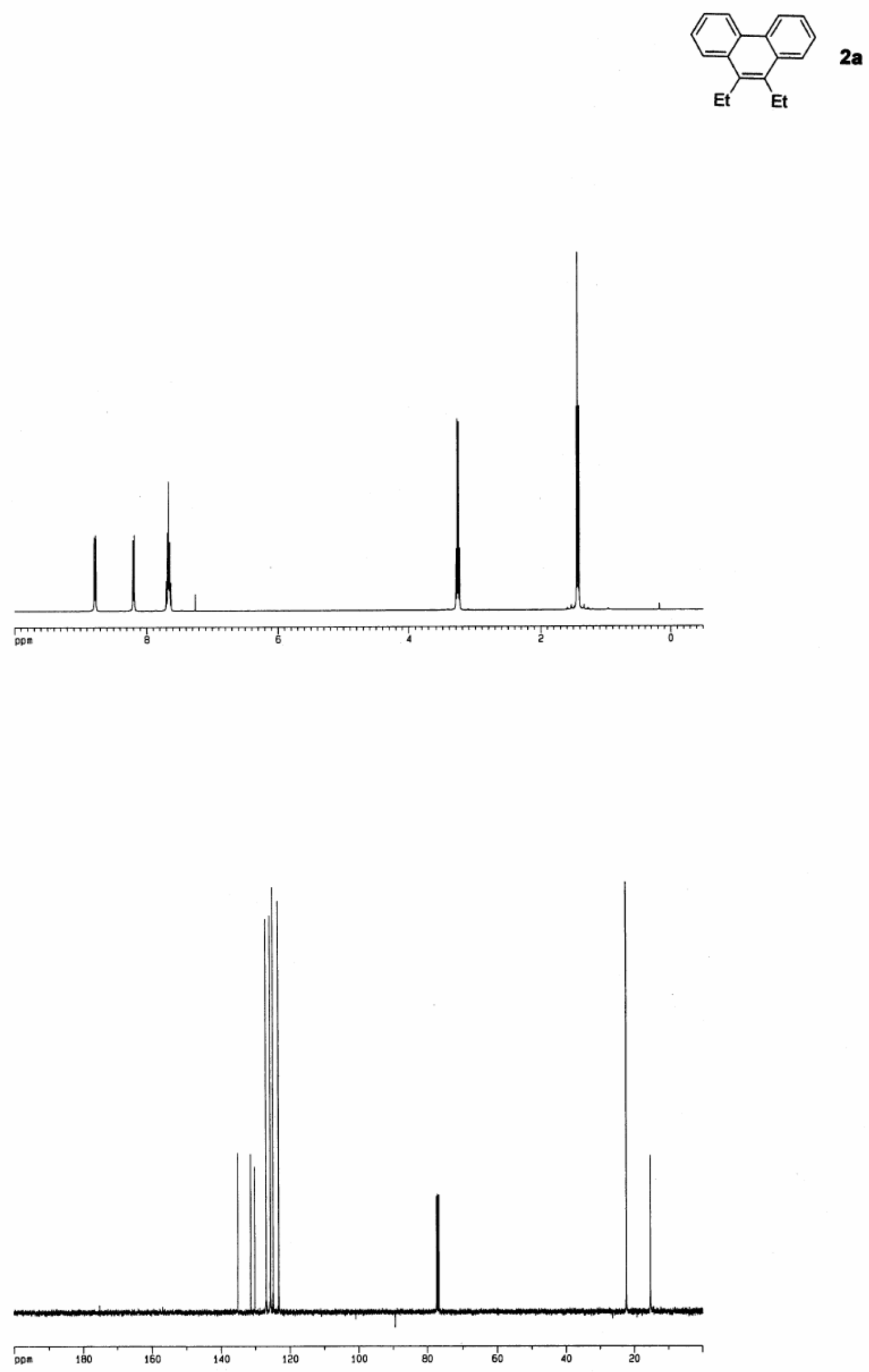

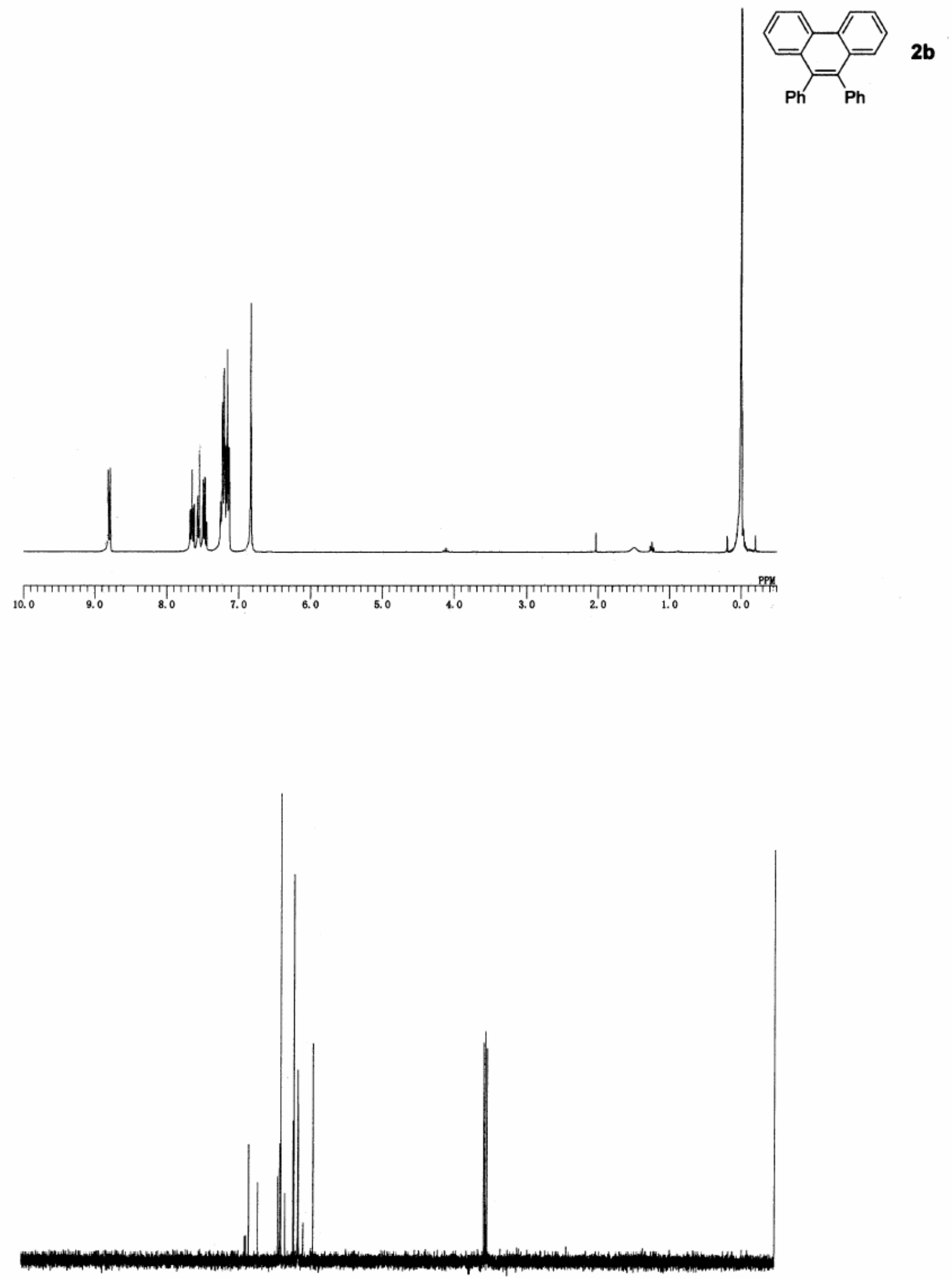

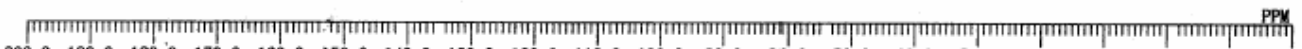
$\begin{array}{lllllllllllllllllllll}200.0 & 190.0 & 180.0 & 170.0 & 160.0 & 150.0 & 140.0 & 130.0 & 120.0 & 110.0 & 100.0 & 90.0 & 80.0 & 70.0 & 60.0 & 50.0 & 40.0 & 30.0 & 20.0 & 10.0 & 0.0\end{array}$ 

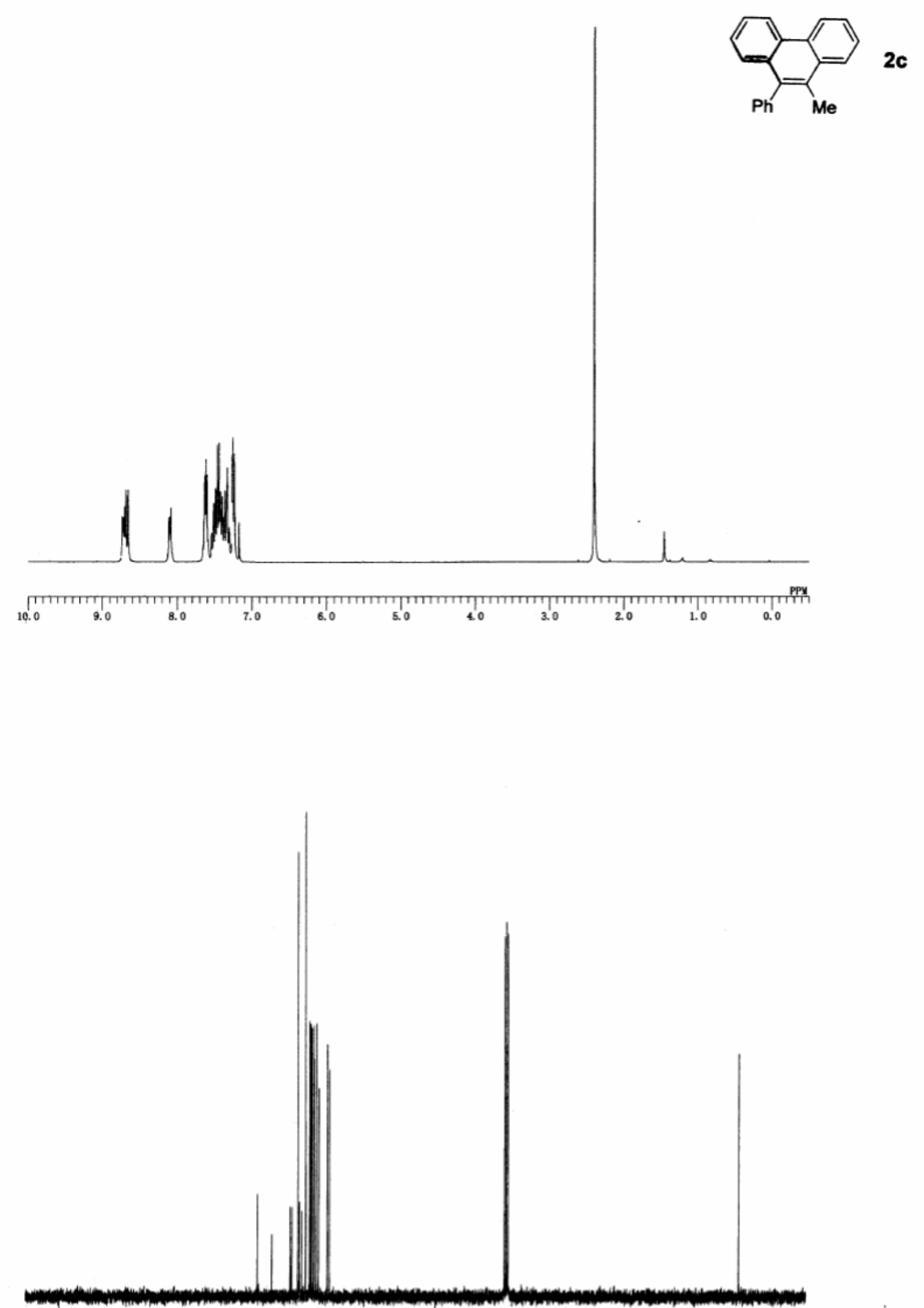

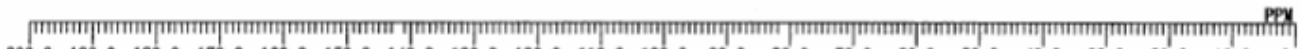
$\begin{array}{lllllllllllllllllllll}200.0 & 190.0 & 180.0 & 170.0 & 180.0 & 150.0 & 140.0 & 130.0 & 120.0 & 110.0 & 100.0 & 90.0 & 80.0 & 70.0 & 00.0 & 50.0 & 40.0 & 30.0 & 20.0 & 10.0 & 0.0\end{array}$ 

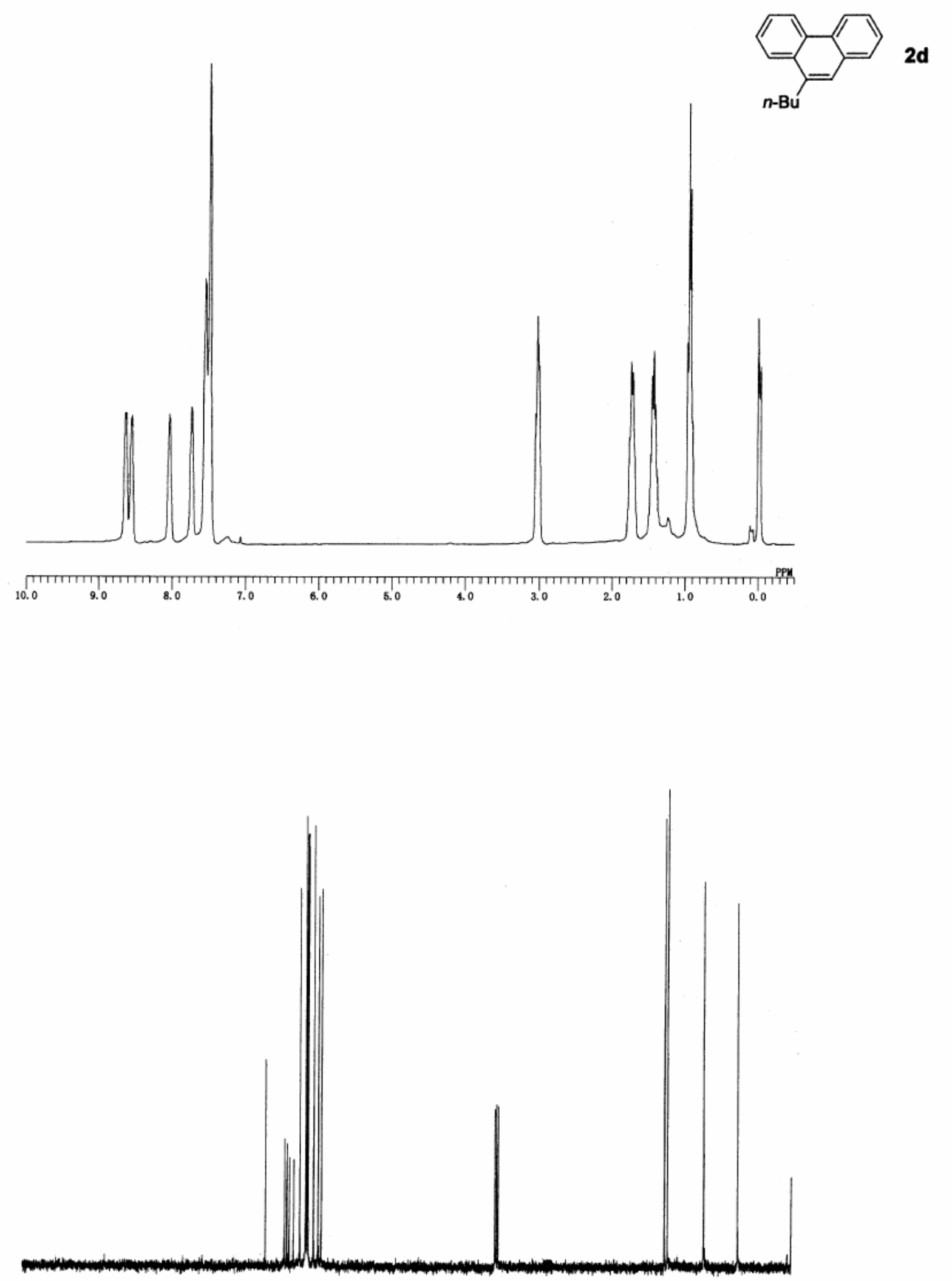

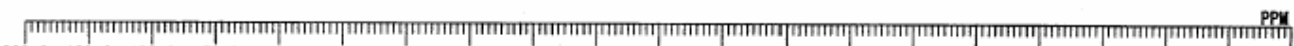

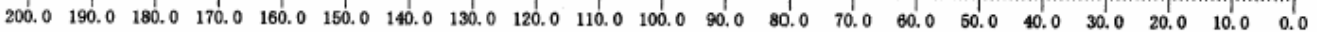



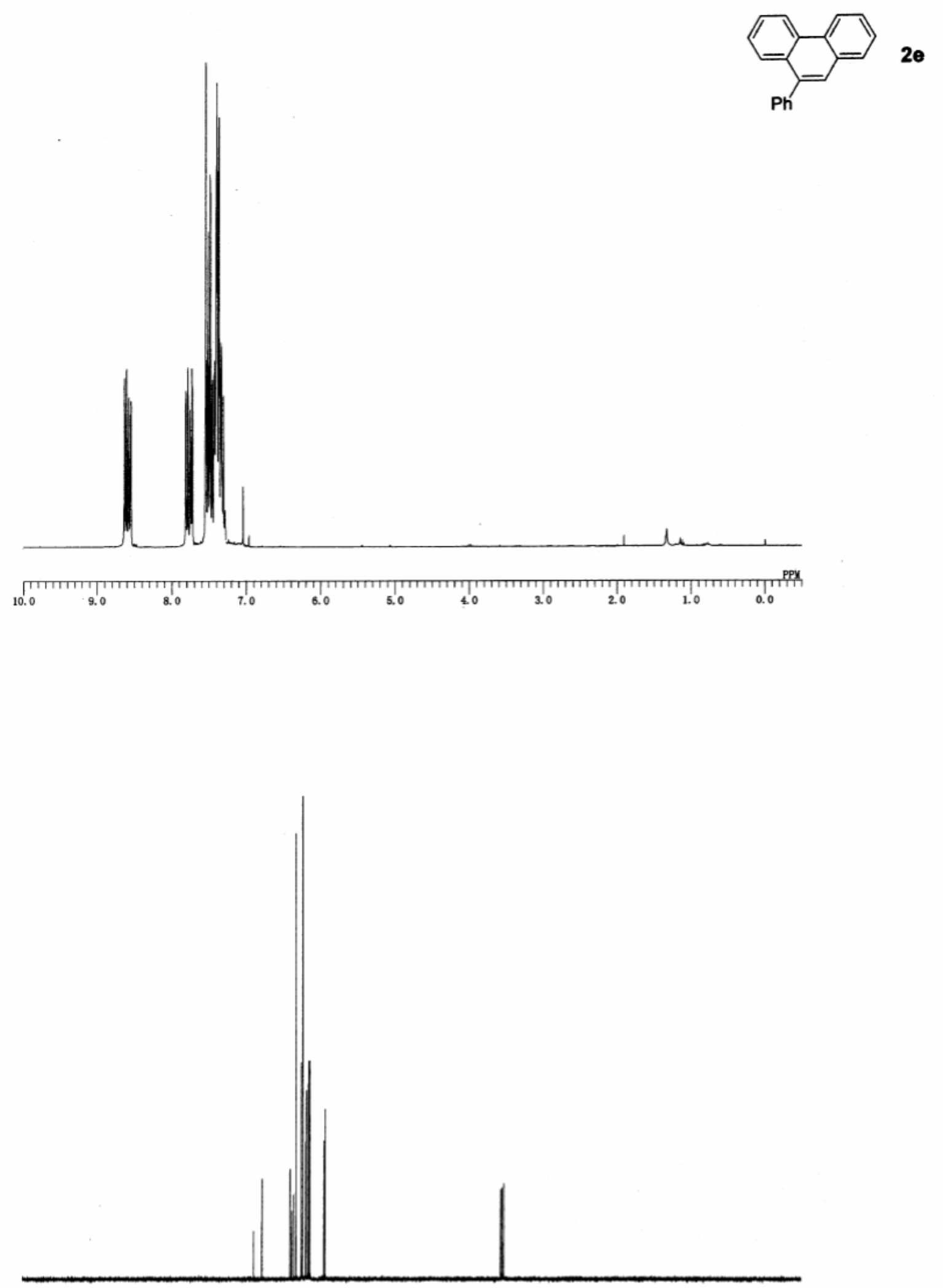

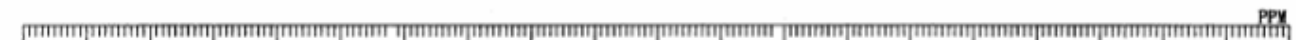
$\begin{array}{lllllllllllllllllllll}200.0 & 190.0 & 180.0 & 170.0 & 160.0 & 150.0 & 140.0 & 130.0 & 120.0 & 110.0 & 100.0 & 90.0 & 80.0 & 70.0 & 60.0 & 50.0 & 40.0 & 30.0 & 20.0 & 10.0 & 0.0\end{array}$ 

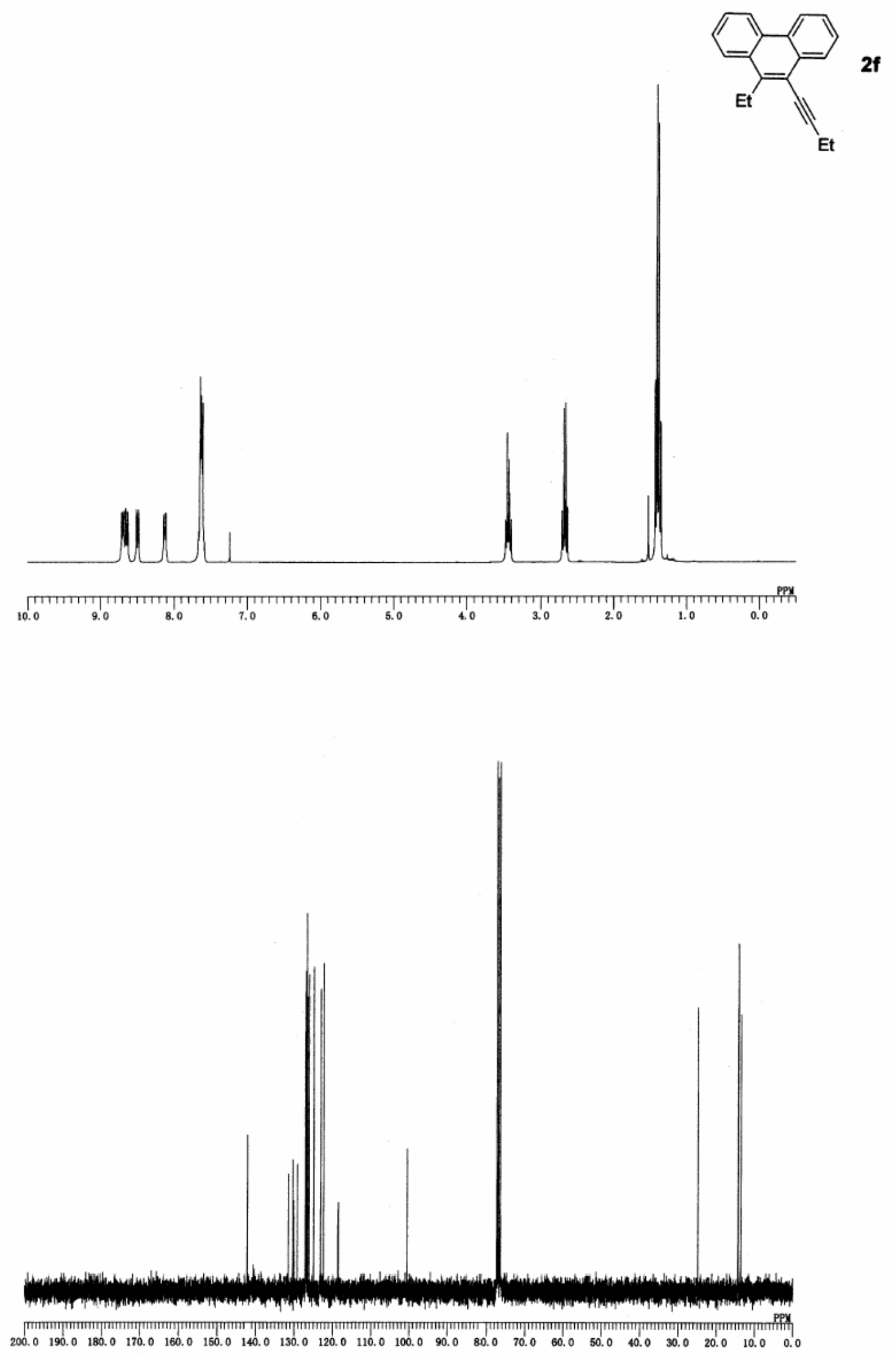

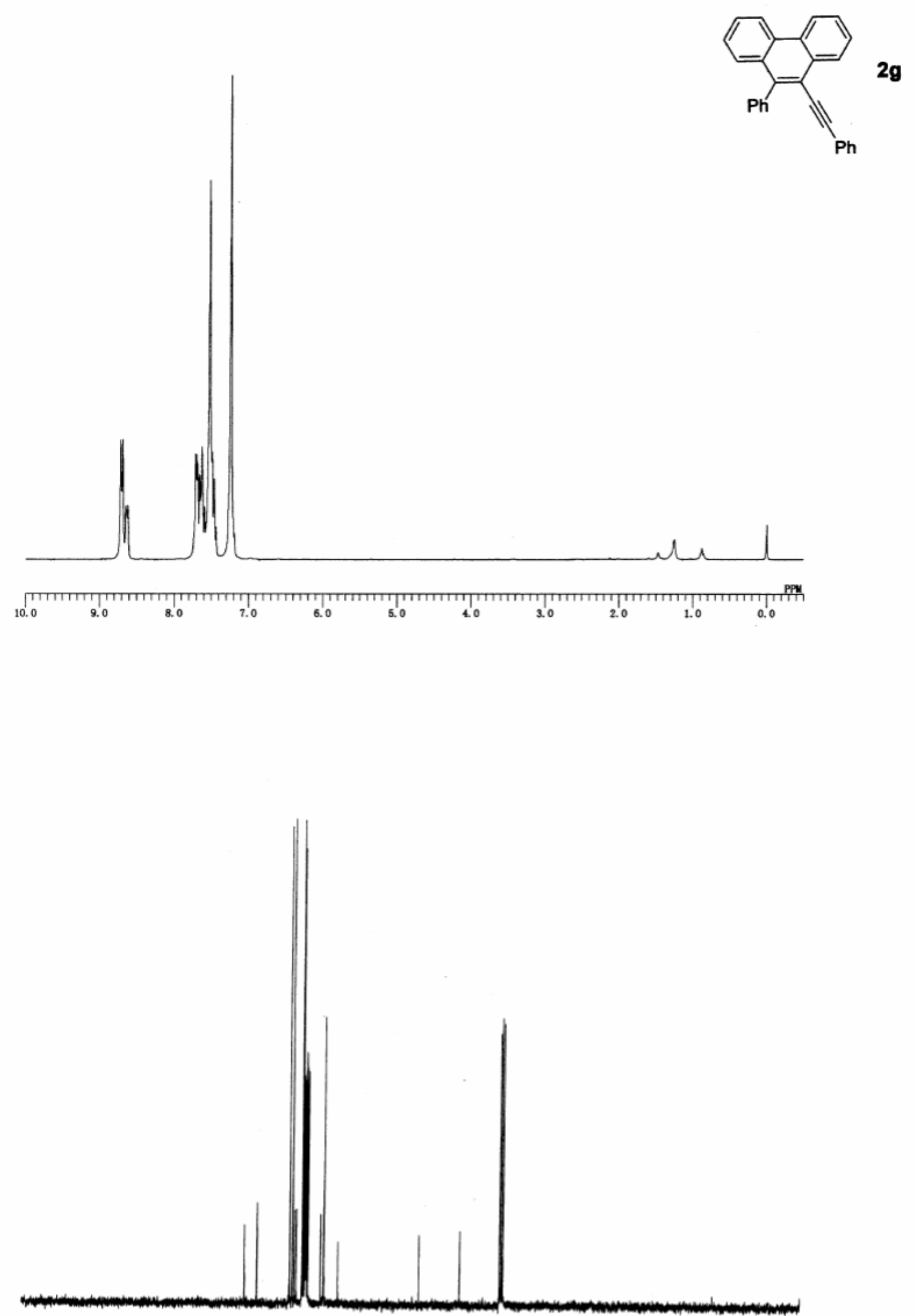

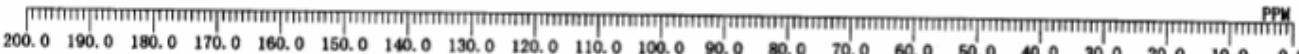




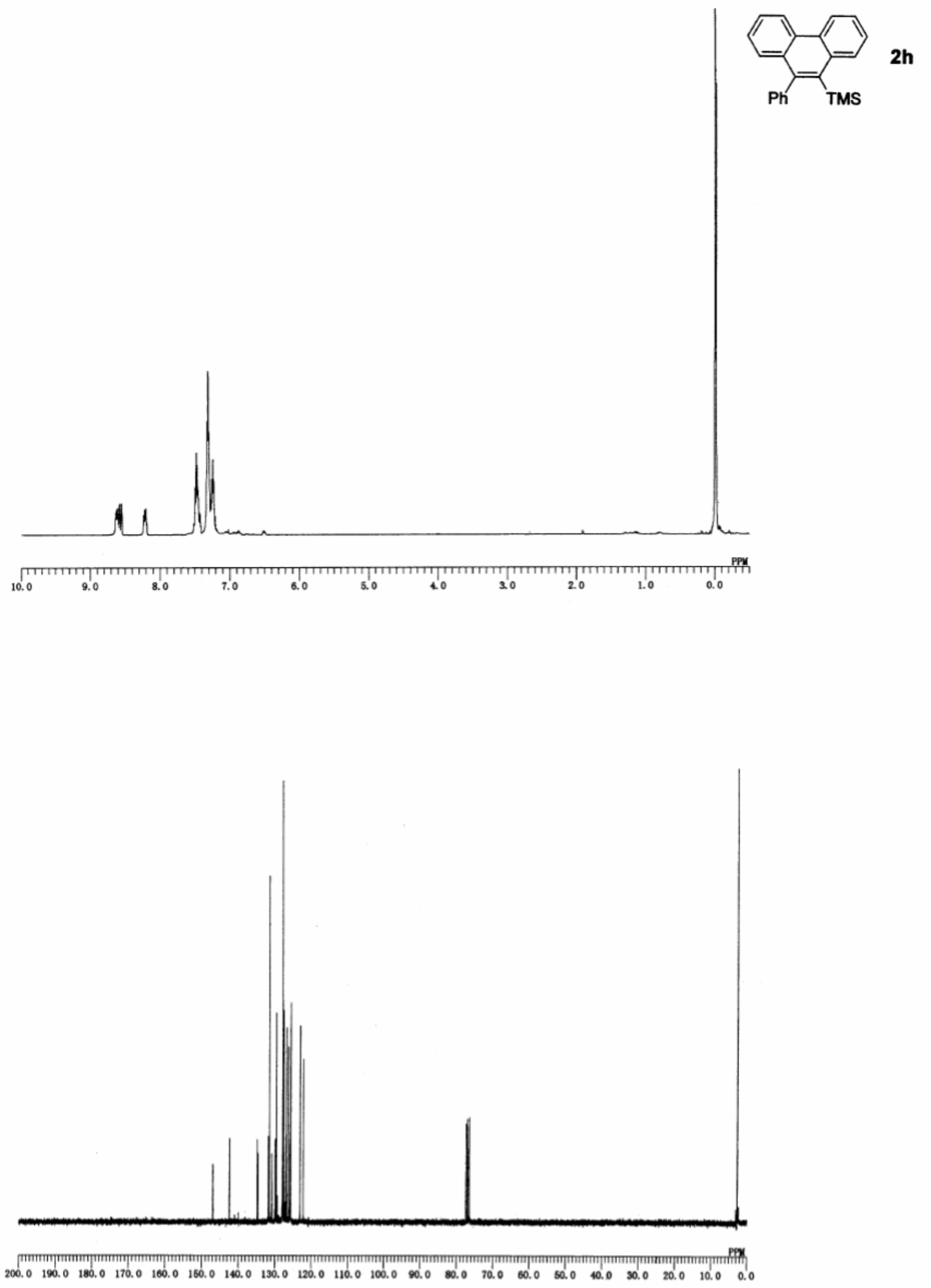



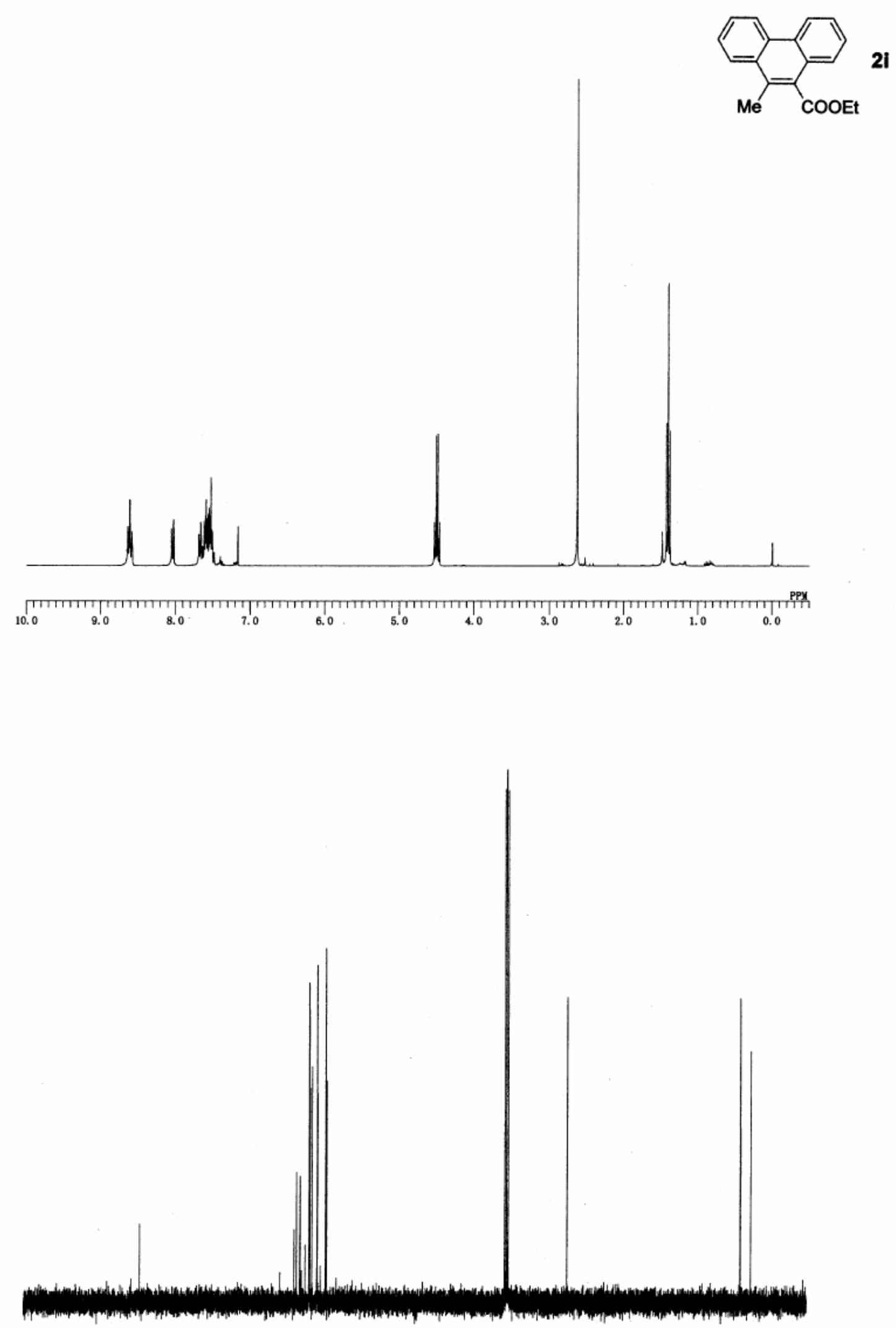

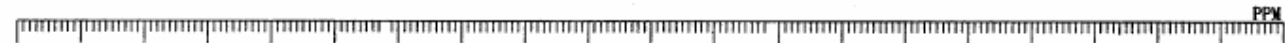
$\begin{array}{lllllllllllllllllllll}200.0 & 190.0 & 180.0 & 170.0 & 160.0 & 150.0 & 140.0 & 130.0 & 120.0 & 110.0 & 100.0 & 90.0 & 80.0 & 70.0 & 60.0 & 50.0 & 40.0 & 30.0 & 20.0 & 10.0 & 0.0\end{array}$ 

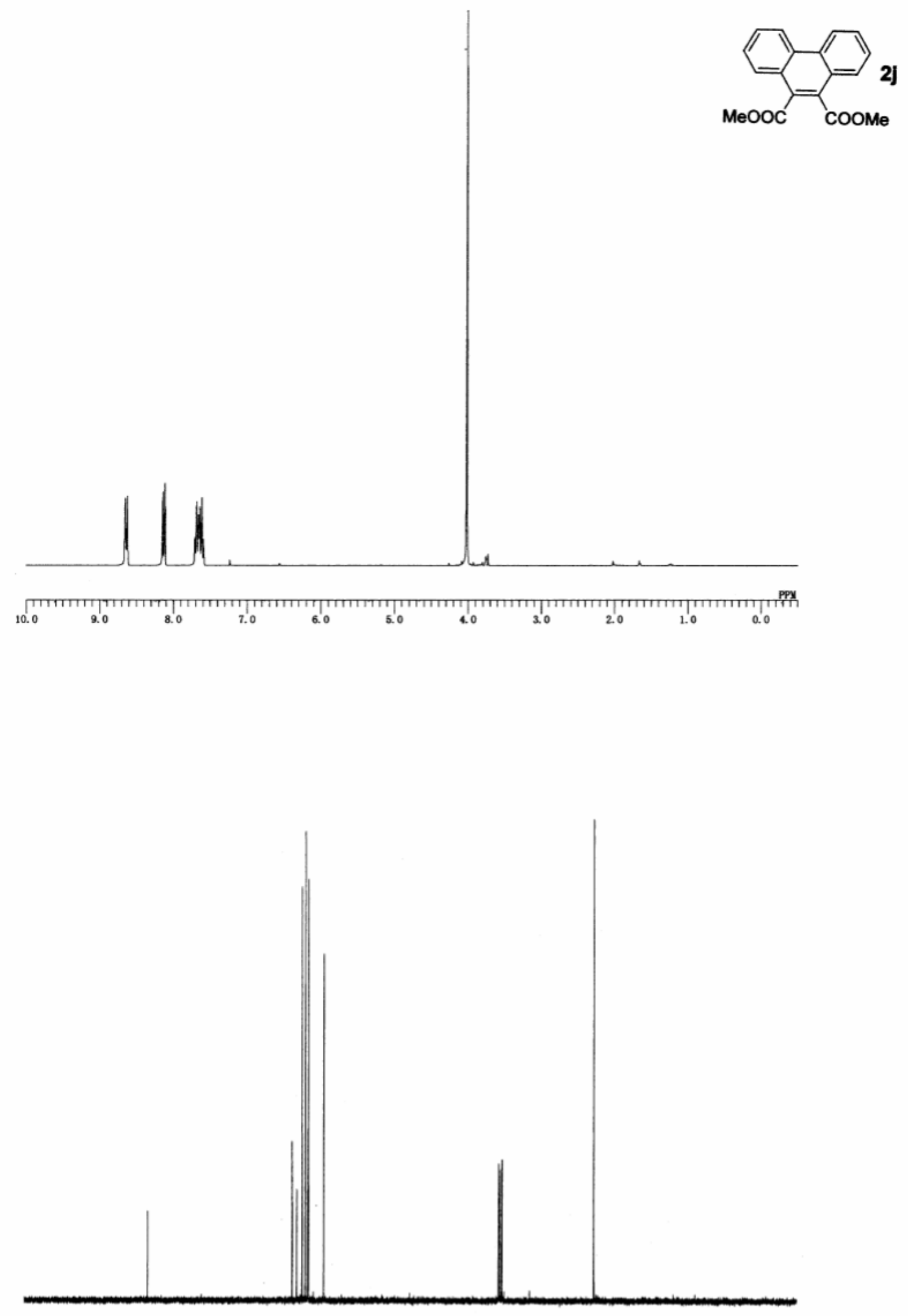

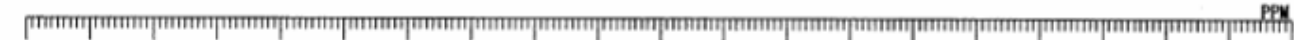

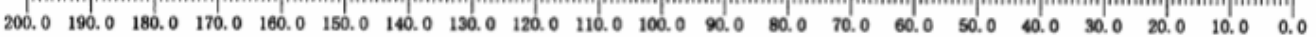



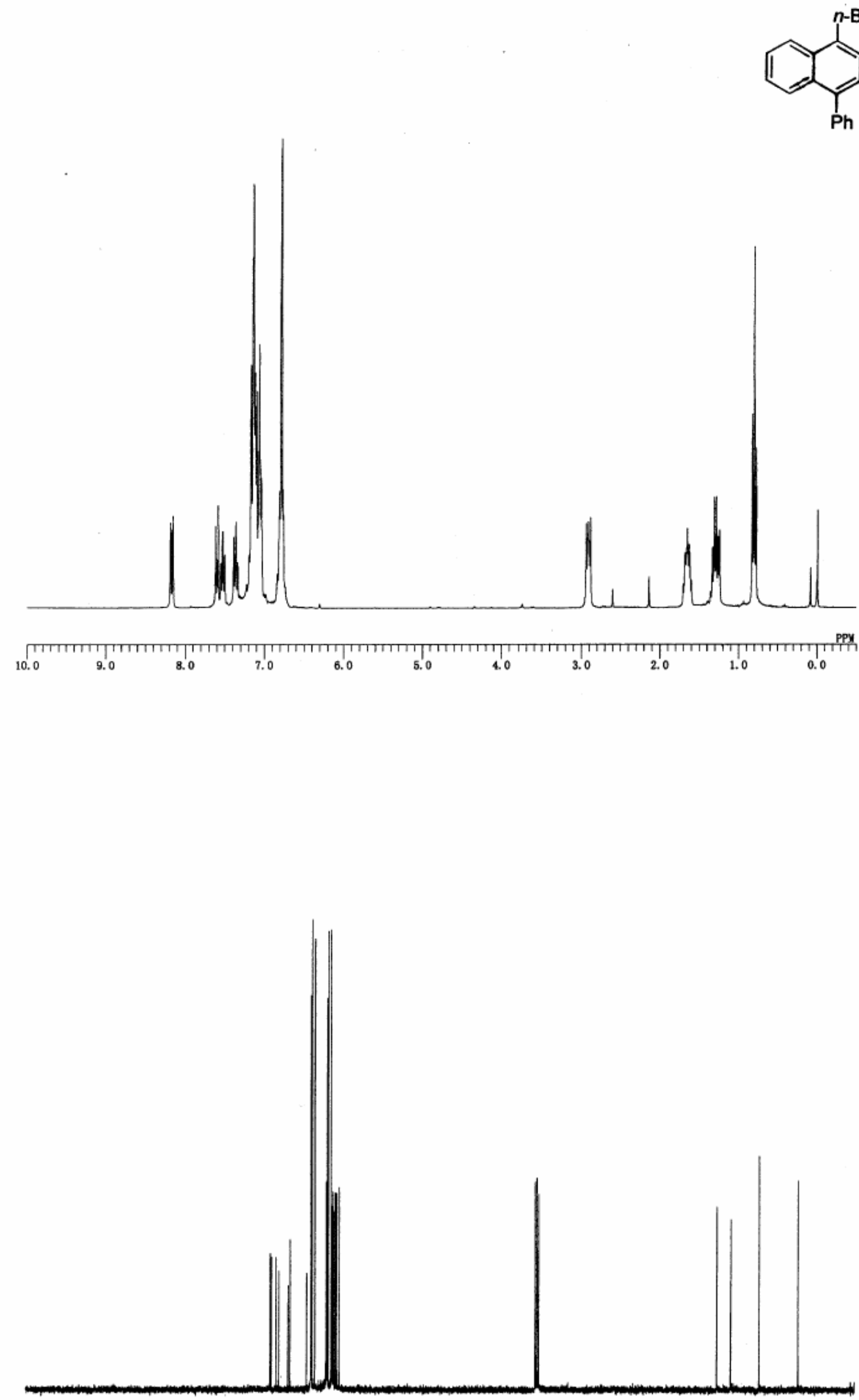

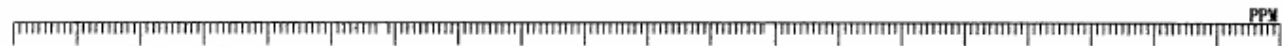
$\begin{array}{lllllllllllllllllllll}00.0 & 190.0 & 180.0 & 170.0 & 160.0 & 150.0 & 140.0 & 130.0 & 120.0 & 110.0 & 100.0 & 90.0 & 80.0 & 70.0 & 60.0 & 50.0 & 40.0 & 30.0 & 20.0 & 10.0 & 0.0\end{array}$ 

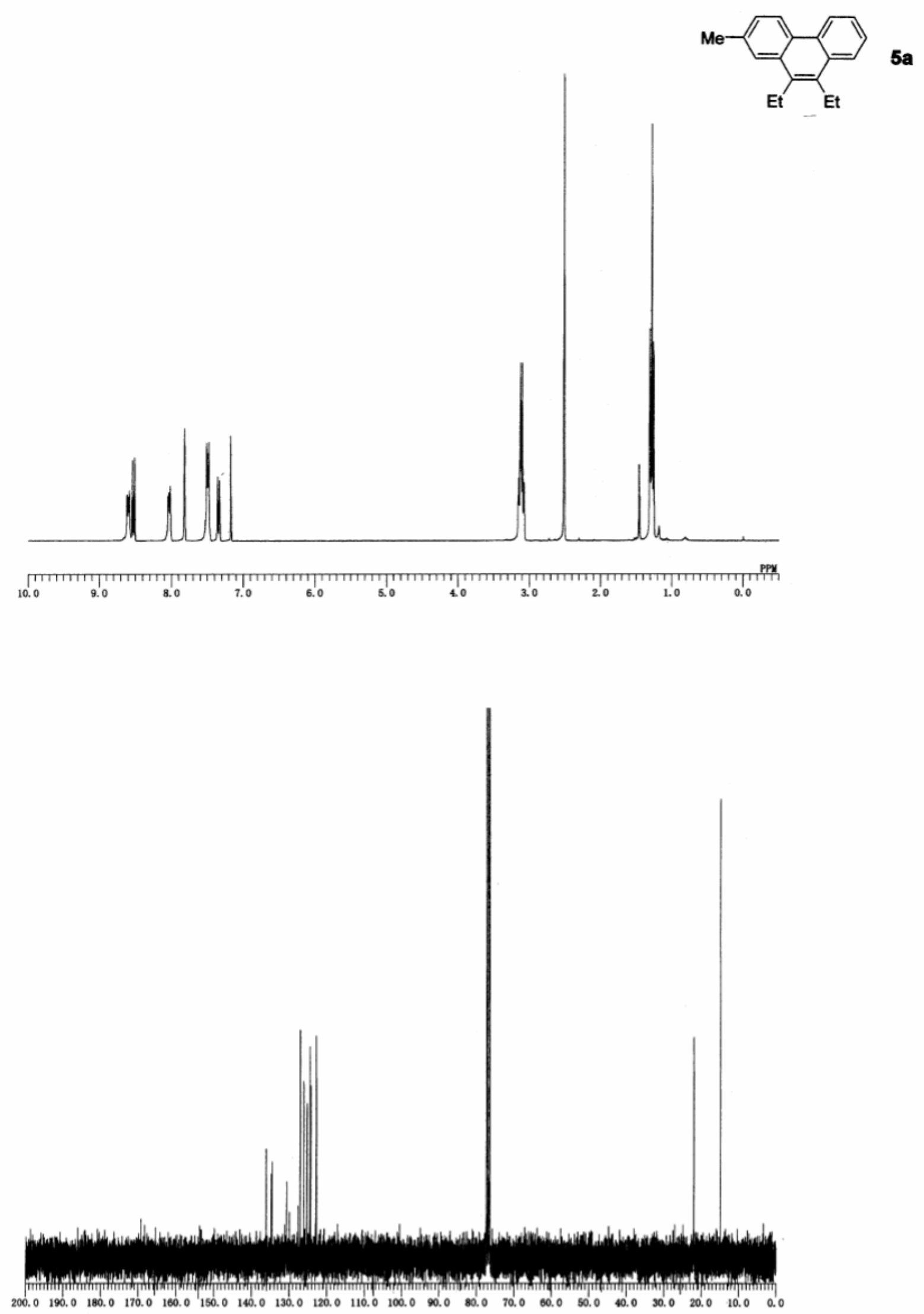

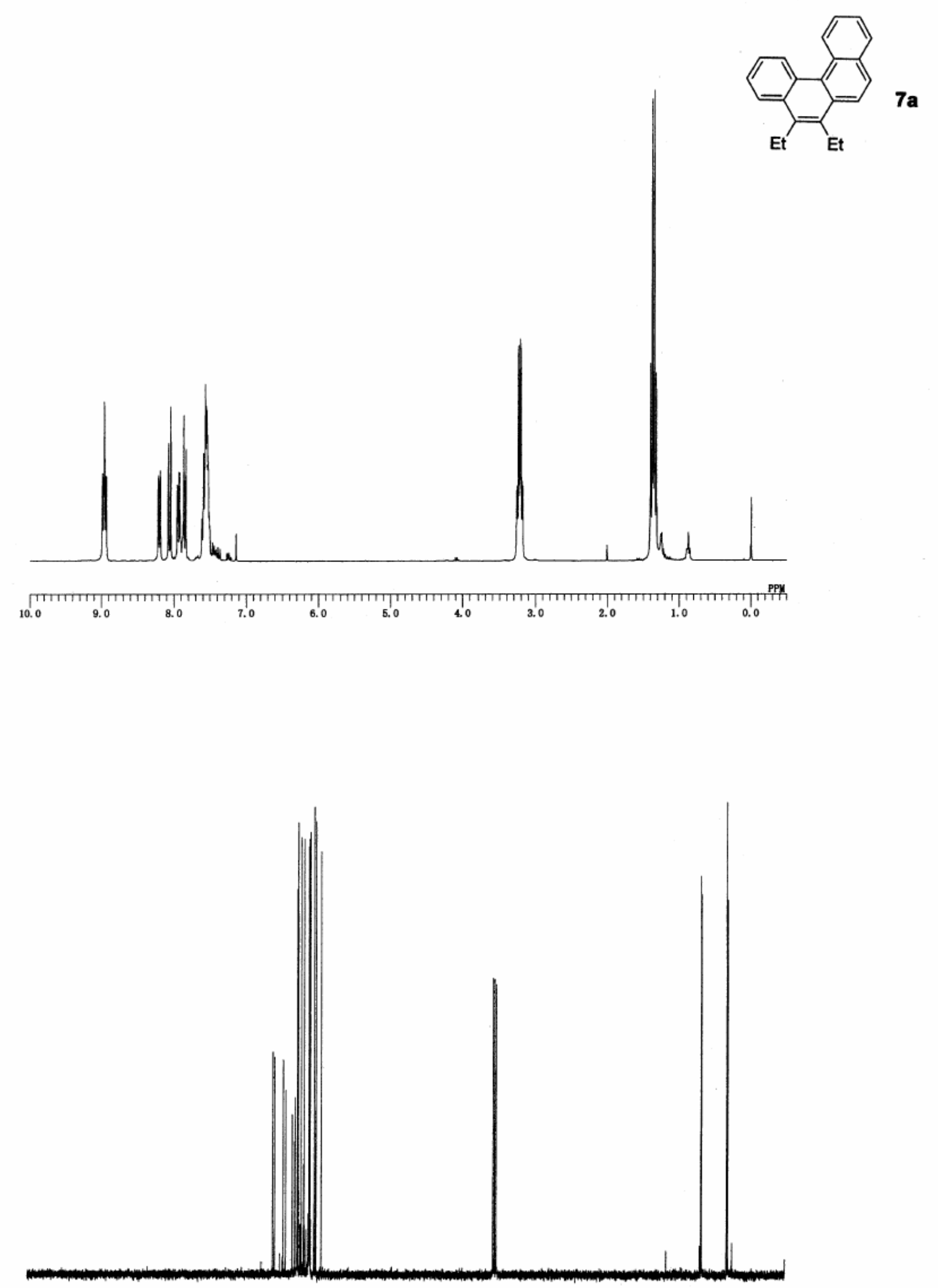

Г $\begin{array}{lllllllllllllllllllll}200.0 & 190.0 & 180.0 & 170.0 & 160.0 & 150.0 & 140.0 & 130.0 & 120.0 & 110.0 & 100.0 & 90.0 & 80.0 & 70.0 & 60.0 & 50.0 & 40.0 & 30.0 & 20.0 & 10.0 & 0.0\end{array}$ 


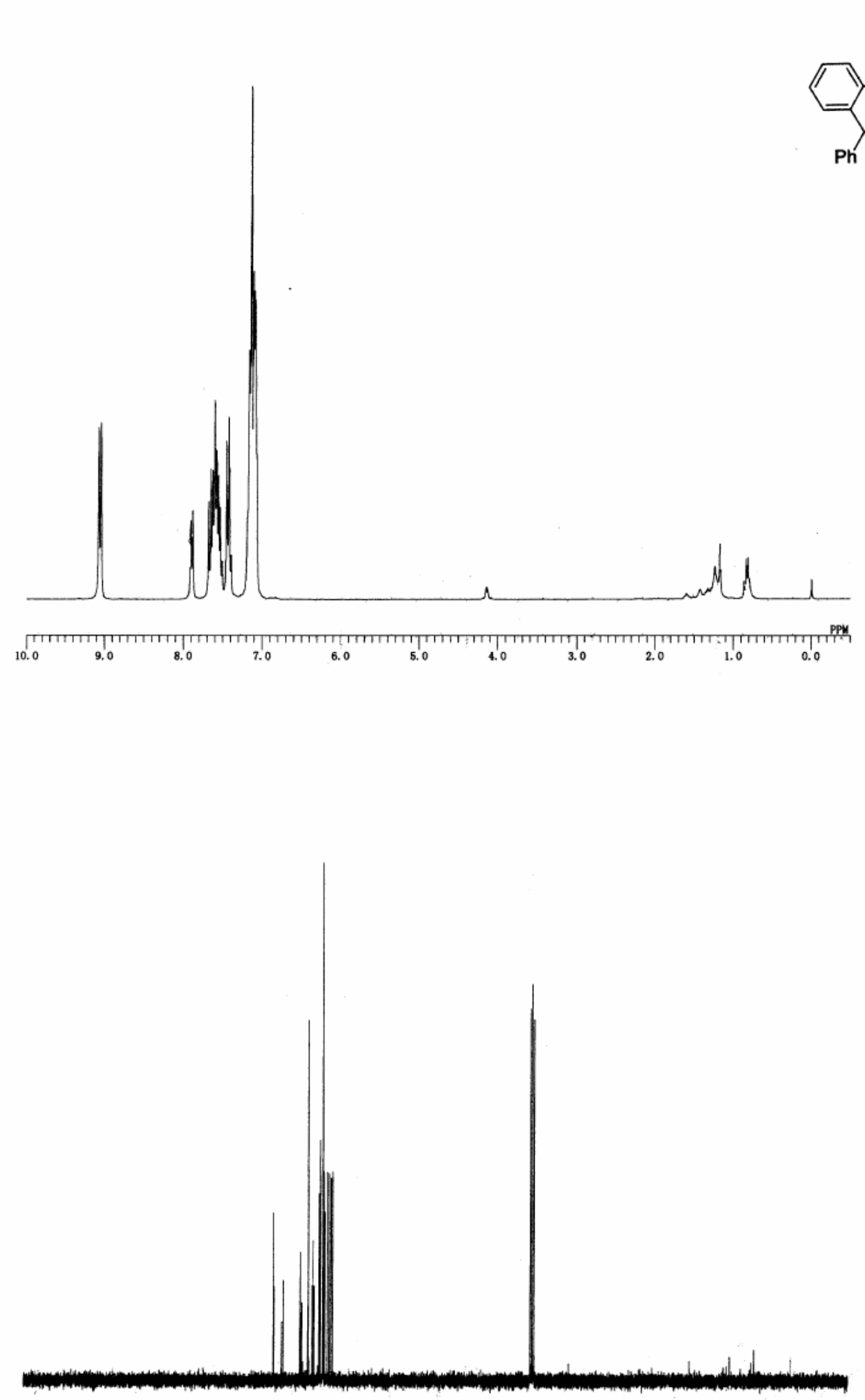

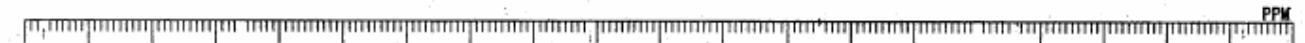

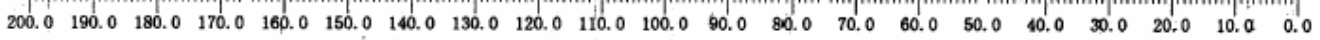



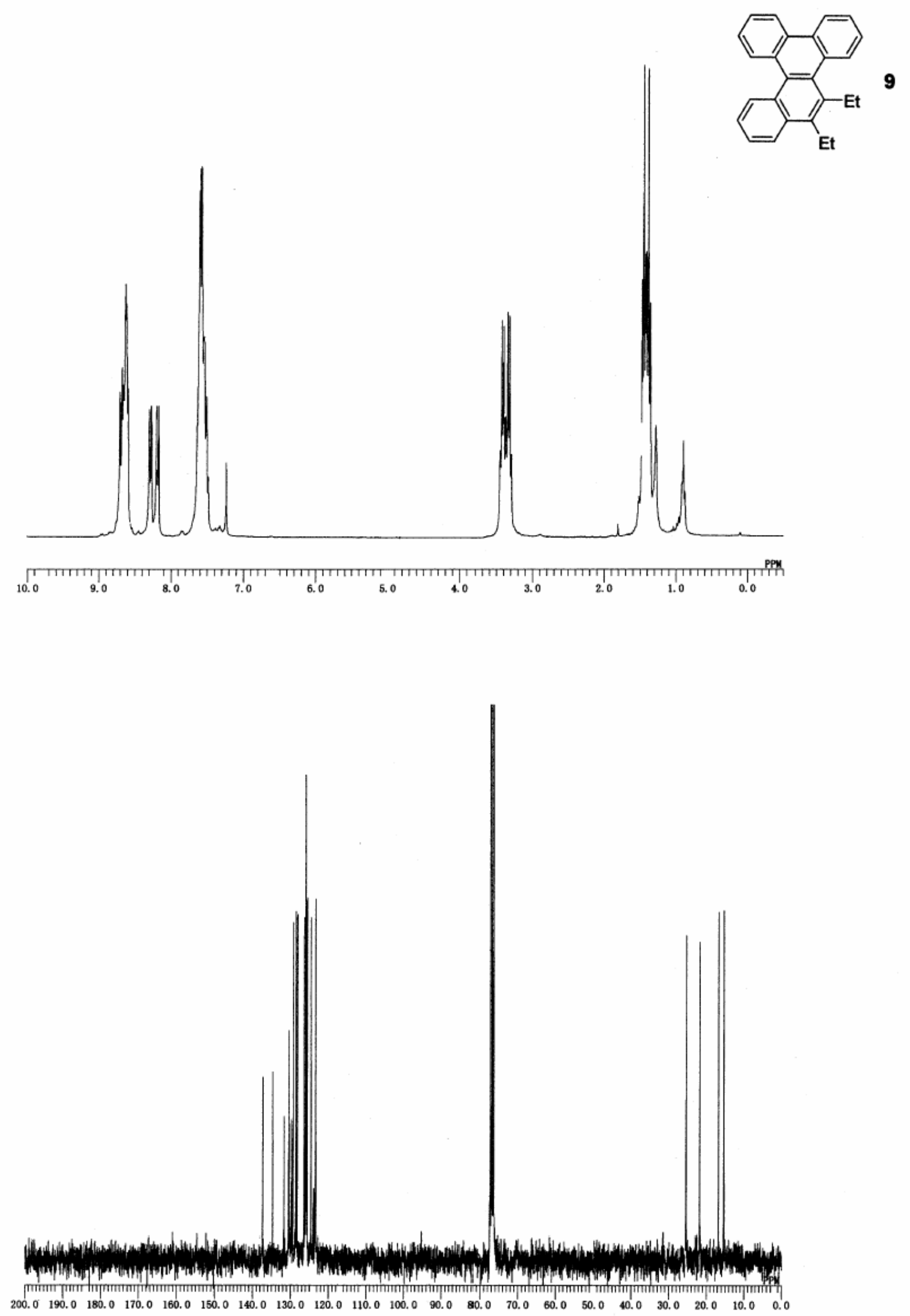


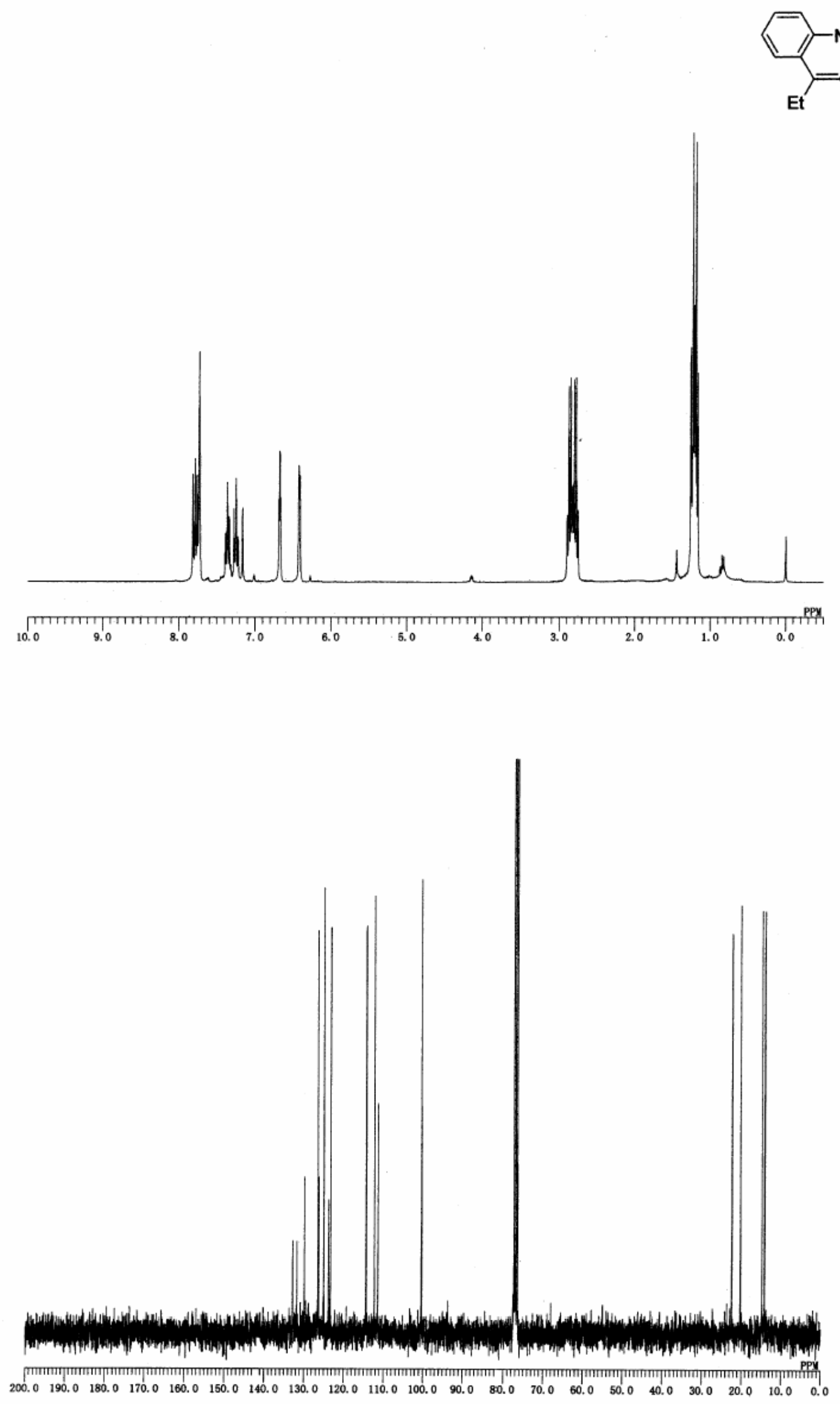



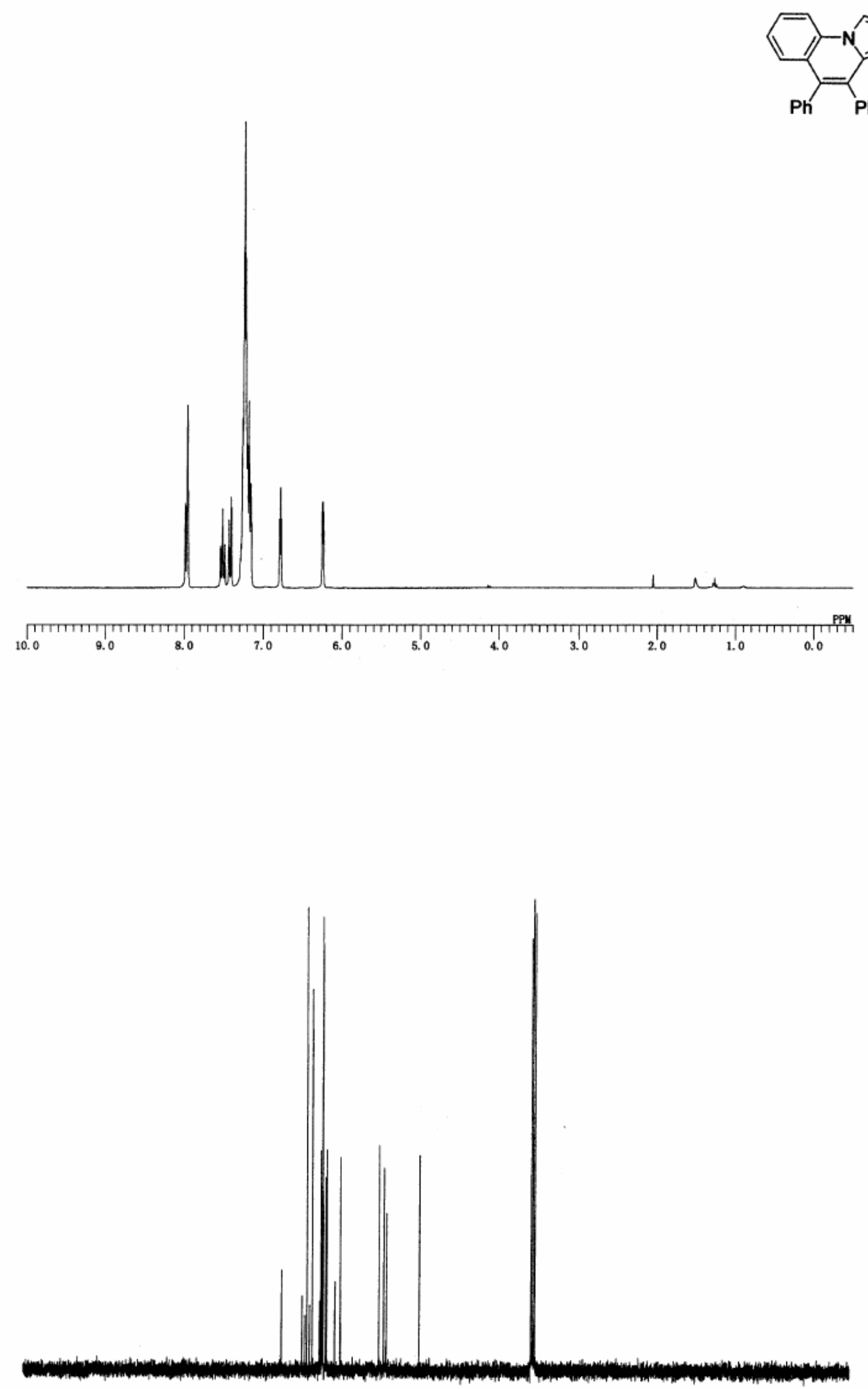

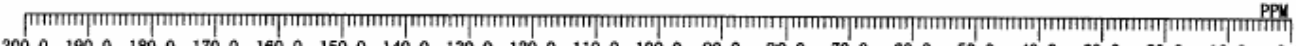

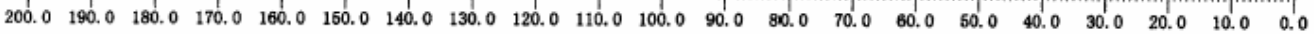



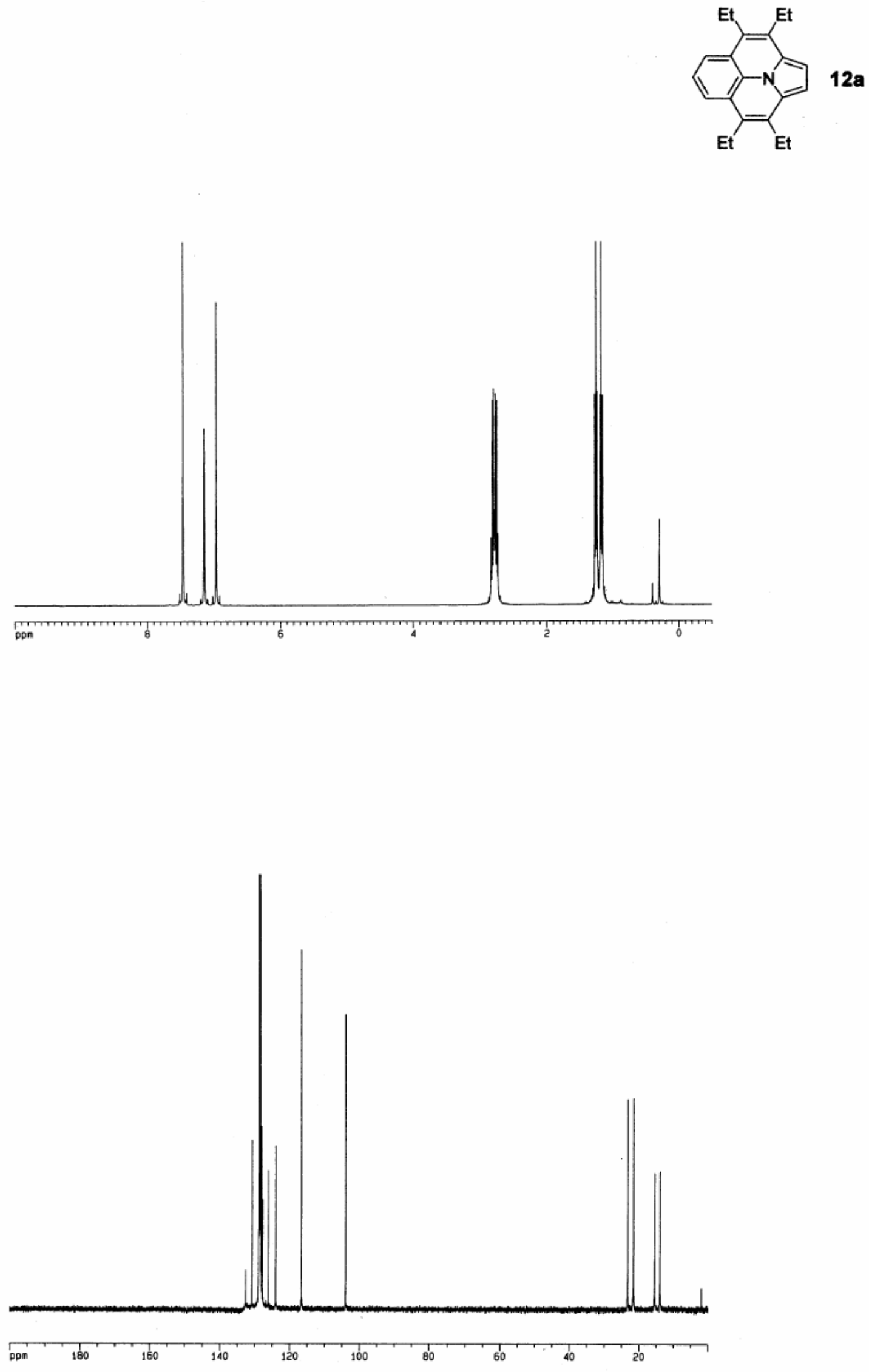

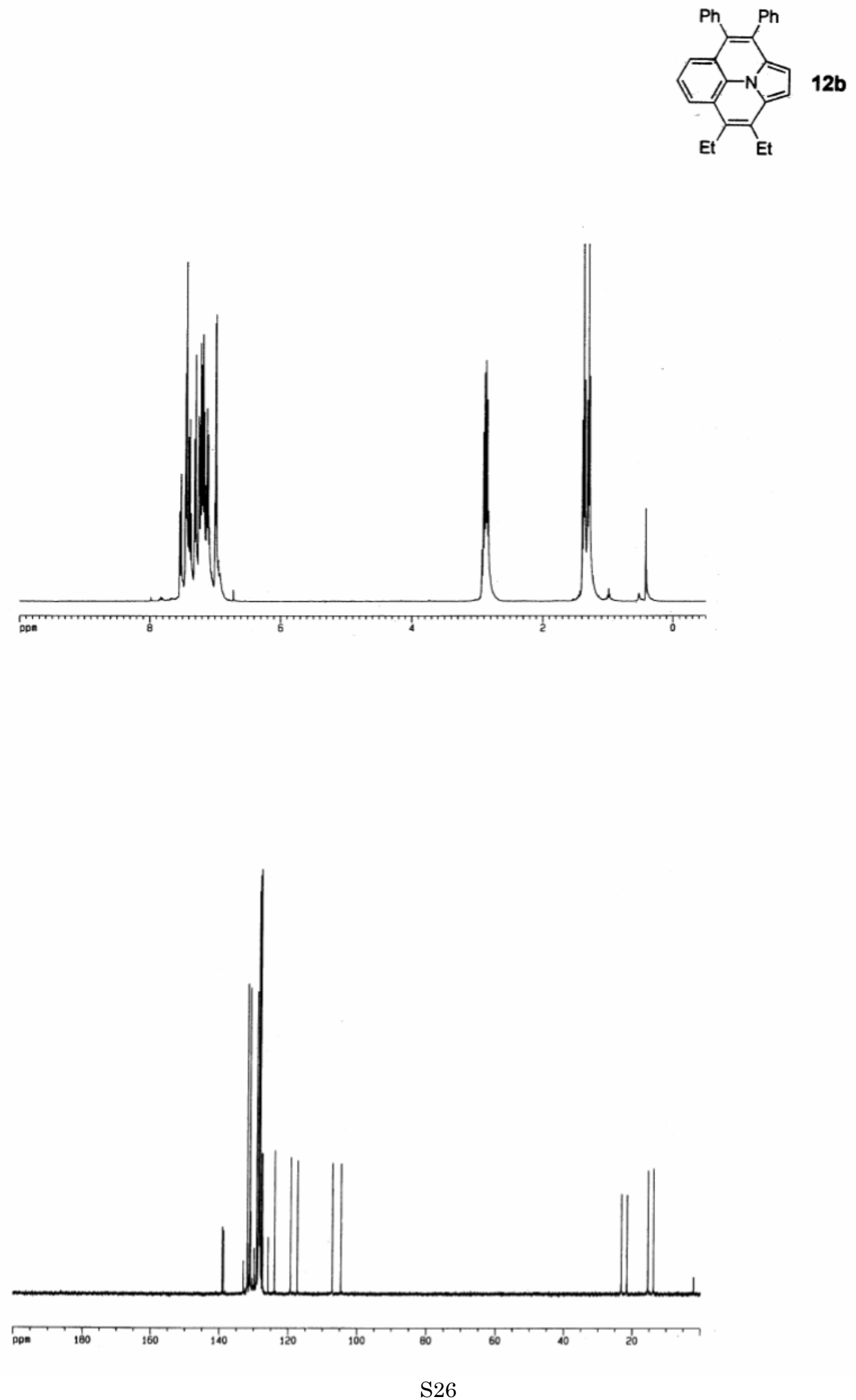

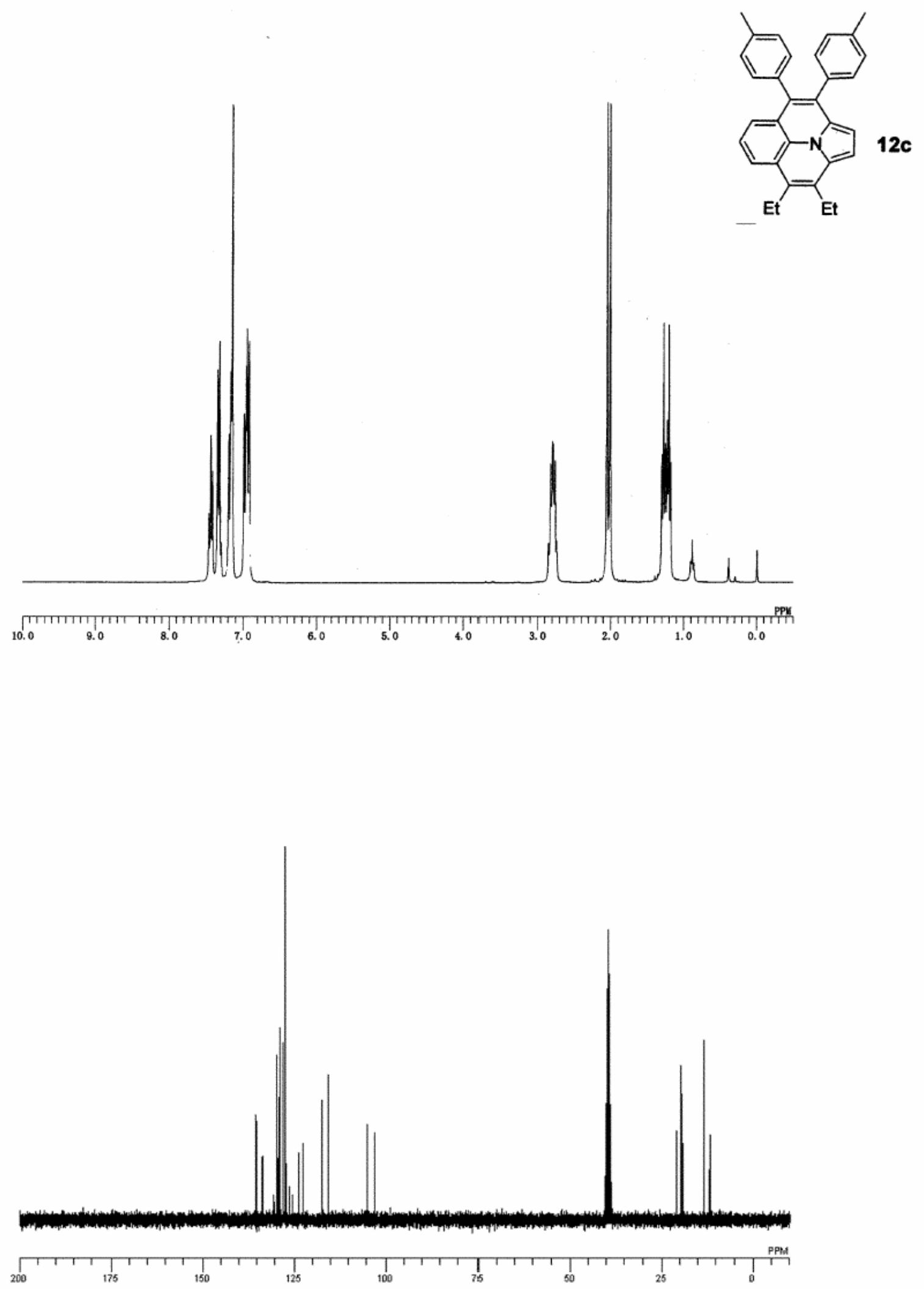

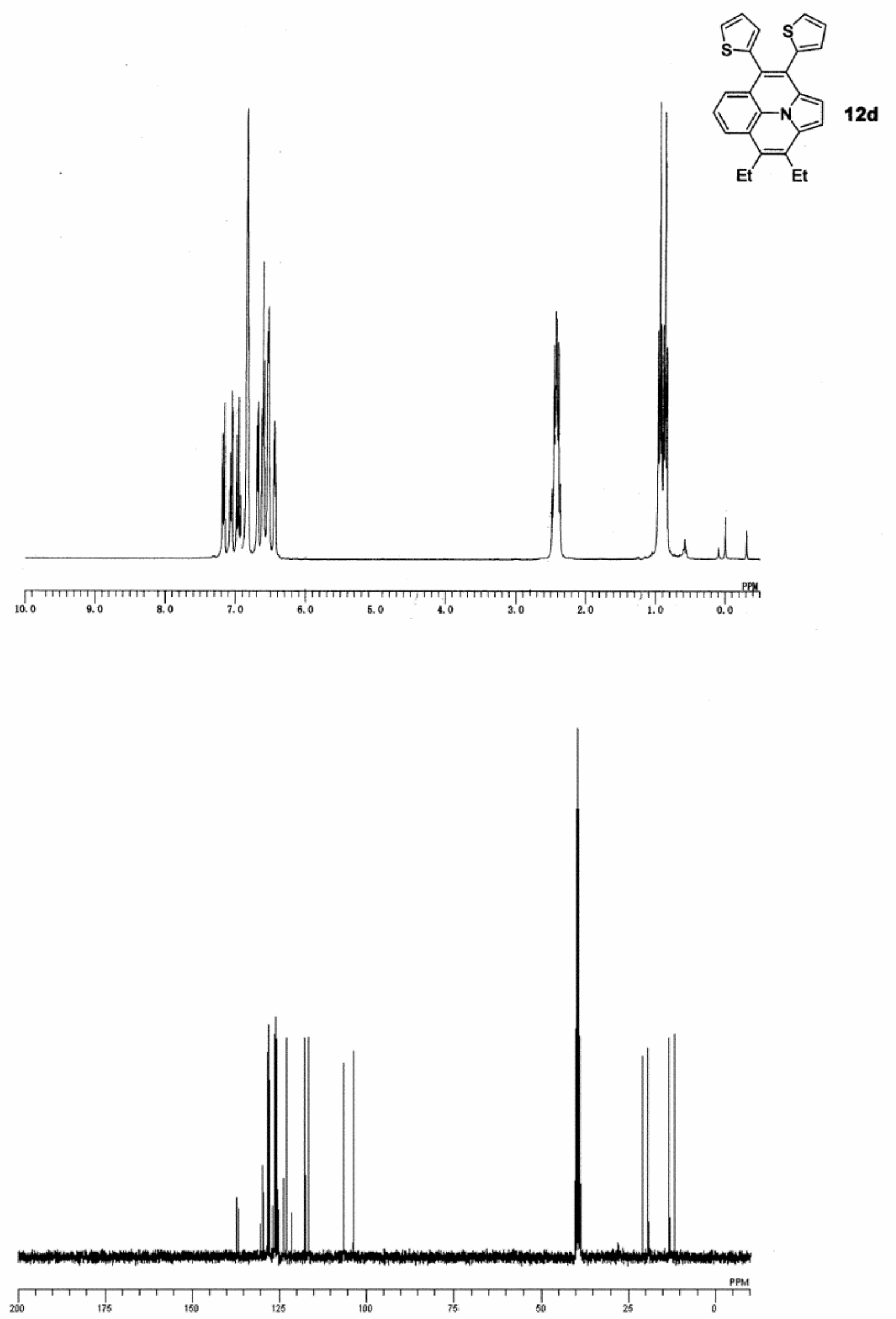